JOURNAL OF THE

AMERICAN MATHEMATICAL SOCIETY

Volume 18, Number 4, Pages 873-918

S 0894-0347(05)00491-1

Article electronically published on June 2, 2005

\title{
THE FAILURE OF RATIONAL DILATION ON A TRIPLY CONNECTED DOMAIN
}

\author{
MICHAEL A. DRITSCHEL AND SCOTT MCCULLOUGH
}

\section{INTRODUCTION}

Let $R$ denote a domain in $\mathbb{C}$ with boundary $B$. Let $X$ denote the closure of $R$.

An operator $T$ on a complex Hilbert space $\mathcal{H}$ has $X$ as a spectral set if $\sigma(T) \subset X$ and

$$
\|f(T)\| \leq\|f\|_{R}=\sup \{|f(z)|: z \in R\}
$$

for every rational function $f$ with poles off $X$. The expression $f(T)$ may be interpreted either in terms of the Riesz functional calculus, or simply by writing the rational function $f$ as $p q^{-1}$ for polynomials $p$ and $q$, in which case $f(T)=p(T) q(T)^{-1}$.

The operator $T$ has a normal $B$-dilation if there exists a Hilbert space $\mathcal{K}$ containing $\mathcal{H}$ and a normal operator $N$ on $\mathcal{K}$ so that

$$
f(T)=\left.P_{\mathcal{H}} f(N)\right|_{\mathcal{H}},
$$

for every rational function $f$ with poles off the closure of $R$. Here $P_{\mathcal{H}}$ is the orthogonal projection of $\mathcal{K}$ onto $\mathcal{H}$.

It is evident that if $T$ has a normal $B$-dilation, then $T$ has $X$ as a spectral set. The Sz.-Nagy Dilation Theorem implies that the closed unit disk is a spectral set for $T$ if and only if $T$ has a unitary dilation. A deep result of Agler says that if the closure of an annulus $\mathbb{A}$ is a spectral set for $T$, then $T$ has a normal boundary of $\mathbb{A}$-dilation [2. Despite a great deal of effort, there has until recently been little progress in determining whether or not these examples are typical or exceptional for finitely connected spectral sets. The problem may be formulated in terms of contractive representations of the algebra of rational functions on the spectral set. Arveson showed [8] that rational dilation holds precisely when such representations are automatically completely contractive. Hence the problem bears some resemblance to the famous problem of Halmos, solved by Pisier [30], on similarity to a contraction, where the question hinged on deciding whether bounded representations of the disc algebra are completely bounded.

Agler, Harland and Raphael [5] have recently given an example of a triply connected domain $R$ and a $4 \times 4$ matrix with $X$ as a spectral set such that this

Received by the editors April 28, 2004.

2000 Mathematics Subject Classification. Primary 47A25; Secondary 30C40, 30E05, 30F10, 46E22, 47A20, 47A48.

Key words and phrases. Dilations, spectral sets, multiply connected domains, inner functions, Herglotz representations, Fay reproducing kernels, Riemann surfaces, theta functions, transfer functions, Nevanlinna-Pick interpolation.

The first author's research was supported by the EPSRC

The second author's research was supported by the NSF.

(C)2005 American Mathematical Society Reverts to public domain 28 years from publication 
matrix does not have a normal $B$-dilation. In this paper we give a proof that for any bounded triply connected domain with analytic disjoint boundary components, there is an operator $T$ with $X$ as a spectral set which does not have a normal $B$ dilation. It can be shown that $T$ can be taken to be a matrix [27, but the argument does not give a bound on the size. Our proof, in contrast to that of Agler, Harland and Raphael, does not use numerical computations. Nevertheless it borrows freely from and overlaps considerably with $[4,3,5]$; see also [6, 11, 12. We implicitly employ the rank one bundle shifts over $R$ [1, 31, the representation for the corresponding reproducing kernels in terms of theta functions due to Fay [20] and Ball and Clancey [10] (see also [15, [16, 13], 32]), and some elementary compact Riemann surface theory.

For the remainder of the paper we assume $-1<c_{1}<0,0<c_{2}<1$ are two points on the real axis, $0<r_{k}<\min \left|c_{k}\right|,\left|1-c_{k}\right|$, and that $R$ is a region obtained by removing disks with centers $c_{k}$ and radii $r_{k}$ from the unit disk. By [14, any bounded triply connected region with boundary components consisting of disjoint analytic curves is conformally equivalent to an annulus with a disk removed. By scaling and rotating if necessary, we assume that the outer boundary of the annulus is the unit circle and that the center of the removed disk is on the real axis. By choosing a point between the removed disk and the central disk of the annulus and applying a Möbius transformation mapping the unit disk to itself and the chosen point to the origin, we get a region $R$ of the sort described initially. Hence there is no loss of generality in restricting ourselves to such regions. Note that in this case, the boundary $B$ of $R$ consists of three components, $B_{0}=\{|z|=1\}$, $B_{1}=\left\{\left|z-c_{1}\right|=r_{1}\right\}$ and $B_{2}=\left\{\left|z+c_{2}\right|=r_{2}\right\}$. We set $X=R \cup B$.

As it happens, only three parameters are really needed to distinguish conformally distinct triply connected domains, so there is some redundancy in using the four parameters $c_{1}, c_{2}, r_{1}, r_{2}$. However, we will later require that no minimal inner function on our domain have a zero of multiplicity three at 0 (all such functions are normalized to have 0 as a zero). We will show that in choosing the point to move to the origin in going from the annulus with the disk removed to the unit disk with disks on either side of the imaginary axis removed in the previous paragraph, there is a choice that enforces this condition on the zeros of these inner functions (Corollary 2.13).

The main theorem of the paper is the following (see also Agler, Harland and Raphael [5]).

Theorem 1.1. For $R$ a bounded triply connected subset of $\mathbb{C}$ the boundary $B$ of which consists of disjoint analytic curves, there exists a Hilbert space $\mathcal{H}$ and a bounded operator $T$ on $\mathcal{H}$ so that $\bar{R}$ is a spectral set for $T$, but $T$ does not have a normal B-dilation.

Here is the idea of the proof. We assume without loss of generality that $R$ is the unit disk with two smaller disks removed, each centered on the real axis and on opposite sides of the imaginary axis. Let $\mathcal{C}$ denote the cone generated by

$$
\left\{H(z)\left(1-\psi(z) \psi(w)^{*}\right) H(w)^{*}: \psi \in B \mathbb{H}(X), H \in M_{2}(\mathbb{H}(X))\right\},
$$

where $B \mathbb{H}(X)$ is the unit ball in the supremum norm of the space of functions analytic in a neighborhood of $X, M_{2}(\mathbb{H}(X))$ the $2 \times 2$ matrices of functions analytic in a neighborhood of $X$. For $F \in M_{2}(\mathbb{H}(X))$, we set

$$
\rho_{F}=\sup \left\{\rho>0: I-\rho^{2} F(z) F(w)^{*} \in \mathcal{C}\right\} .
$$


It happens that $\rho_{F}>0$. Suppose that $F$ is unitary-valued on $B$ and that $\rho_{F}<1$. Then there exists a Hilbert space $\mathcal{H}$ and an operator $T$ such that $T$ has $X$ as a spectral set, but $T$ does not have a normal $B$-dilation (Theorem 5.2). We also show that if $F \in M_{2}(\mathbb{H}(X))$ is as above and $\rho_{F}=1$, then under suitable assumptions regarding zeros, $F$ has a Herglotz representation (Theorem [5.10). The kernel of the adjoint of multiplication by $F$ in this case has its kernel spanned by a finite collection of reproducing kernels due to Fay (a variant on the Szegö kernel for harmonic measure - Lemma 5.11), and this results in $F$ being diagonalizable (Theorem [5.13). Our counterexample is obtained by constructing a function satisfying the boundary conditions and hypotheses concerning zeros mentioned above which is not diagonalizable. For such a function, $\rho_{F}<1$, and hence rational dilation fails.

The organization of the paper differs somewhat from the outline of the proof mentioned above. Minimal inner functions play a key role throughout, so we begin by detailing the results on harmonic functions and analytic functions with positive real parts which are related to these inner functions via a Cayley transform. Much of this material can be found in Grunsky's monograph 22 for more general planar domains. These inner functions are used to give a "scalar" Herglotz representation theorem for functions analytic in a neighborhood of $R$ with unimodular boundary values (Proposition 2.14). The reader is also referred to [5], where the importance of Herglotz representations in characterizing spectral sets is cogently presented.

We then seemingly digress into some basic results on Riemann surfaces and theta functions, which are useful in constructing meromorphic functions on a compact Riemann surface with a given zero/pole structure. The compact surface $Y$ we consider is very special: it is the double of our two-holed region $R$, obtained topologically by gluing a second copy of $R$ to itself along $B$. Our minimal inner functions extend to meromorphic functions on the double via reflection, and indeed the same goes for matrix-valued inner functions.

Using fairly elementary tools, we are able to say quite a bit about the zero structure of the minimal inner functions on $R$ based on the parametrization of these functions. We then extend results on scalar inner functions to some $2 \times 2$ matrixvalued inner functions, constructing a family of such functions which will ultimately give rise to our example of such a function with a particular zero structure which is not diagonalizable.

We then turn to considering Szegö kernels $K^{a}(\zeta, z)$ on $R$ with respect to harmonic measure for the point $a \in R$. The truly remarkable fact discovered by Fay 20 is that these kernels extend to meromorphic functions on the double $Y$. Indeed, Fay gives a representation for $K^{a}(\zeta, z)$ in terms of theta functions and the prime form. Ball and Clancey [10] give a similar representation, which is actually a bit more explicit as it only involves theta functions for the $n$-torus family of Abrahamse kernels associated to a multiply connected domain of connectivity $n+1$. The explicit nature of the theta function representation of the meromorphic kernels $K^{a}(\zeta, z)$ allows the determination of their zero/pole structure. Next we consider certain finite linear combinations of $K^{a}(\zeta, z)$ with coefficients in $\mathbb{C}^{2}$, and show that if an analytic function is in the span of certain kernels, then it must be constant. This is used later to prove the diagonalization result mentioned above.

We next turn to representing nice $2 \times 2$ matrix-valued inner functions-these are functions which are unitary valued on the boundary with what we term a "standard" zero set - see section 3.2 for the definition. First we show the connection 
between the failure of rational dilation and $\rho_{F}$ being strictly less than one for some contractive analytic function $F$ with unitary boundary values. We make a brief foray into matrix measures, and then prove that when $\rho_{F}=1$ for such functions, we have a nice representation for $1-F(z) F(w)^{*}$. Part of this relies on an Agler-Nevanlinna-Pick interpolation result (Proposition 5.8), proved using a transfer function representation, as well as a uniqueness result (Proposition 5.9). First we show that a Herglotz type representation holds over finite subsets of points in $R$ for such an $F$. This is then extended to all of $R$ via the interpolation theorem, and if the finite set of points was chosen in just the right way, this extension is unique. The result is what we term a "tight" representation.

In the next section we return to Fay's kernel, and use it to prove our diagonalization result. We show that some of the matrix inner functions constructed earlier are not diagonalizable, proving our main theorem.

The last section shows why, once we know that rational dilation fails, there is a finite-dimensional example. This part is based on work of Paulsen [27].

\section{Spectral Sets And some FunCtion theORY on $R$}

We begin with some standard material on spectral sets. Then we review harmonic functions, analytic functions of positive real part, and inner functions on $R$, the unit disk with two disks removed as described above.

2.1. Spectral sets. Let $\mathbb{H}(X)$ denote complex-valued functions which are analytic in a neighborhood of $X$, and $M_{2}(\mathbb{H}(X))$ the $2 \times 2$ matrices of such functions. We likewise let $\mathcal{R}(X)$ denote the rational functions with poles off of $X$, with $M_{2}(\mathcal{R}(X))$ defined in the obvious manner. For $f$ in either $\mathbb{H}(X)$ or $M_{2}(\mathbb{H}(X))$ define

$$
\|f\|=\|f\|_{R}=\sup \{\|f(z)\|: z \in R\}
$$

where $\|f(z)\|$ is the modulus of the scalar $f(z)$ or the operator norm of the $2 \times 2$ matrix $f(z)$ respectively.

Throughout the rest of this section $T$ is a bounded operator on the complex Hilbert space $\mathcal{H}$ which has $X$ as a spectral set.

To the operator $T$ associate the homomorphism, $\phi_{T}: \mathcal{R}(X) \rightarrow \mathcal{B}(\mathcal{H})$ by $\phi_{T}(p / q)$ $=p(T) q(T)^{-1}, p$ and $q$ polynomials. When $T$ has a normal $B$-dilation, equation (1.1) can then be expressed as $\phi_{T}(f)=P_{\mathcal{H}} f(N) \mid \mathcal{H}$ for $f \in \mathcal{R}(X)$. Using the Riesz functional calculus and Runge's theorem, the map $\phi_{T}$ extends continuously to $\phi_{T}$ : $\mathbb{H}(X) \rightarrow \mathcal{B}(\mathcal{H})$. Conversely, a contractive unital homomorphism $\pi: \mathbb{H}(X) \rightarrow \mathcal{B}(\mathcal{H})$, that is, a unital homomorphism satisfying

$$
\|\pi(f)\| \leq\|f\|_{R}
$$

for $f \in \mathbb{H}(X)$, determines an operator with $X$ as a spectral set.

If $T$ has a normal $B$-dilation as in (1.1) and if $G$ is in $M_{2}(\mathcal{R}(X))$, then

$$
G(T)=P_{\mathcal{H} \oplus \mathcal{H}} G(N) \mid \mathcal{H} \oplus \mathcal{H}
$$

Since, by the maximum principle, $\|G(N)\|=\|G\|_{R}$, it follows that

$$
\|G(T)\| \leq\|G\|_{R} .
$$

Indeed, the same reasoning implies this for any $G \in M_{n}(\mathcal{R}(X))$, or equivalently, that $\phi_{T}$ is completely contractive. 
If $F \in M_{2}(\mathbb{H}(X))$ (so that the entries $F_{j, \ell}$ of $F$ are analytic in a neighborhood of $X$ ), but not necessarily rational, it then still makes sense to consider, on $\mathcal{H} \oplus \mathcal{H}$, the operator

$$
F(T)=\left(F_{j, \ell}(T)\right)
$$

where the $F_{j, \ell}(T)$ are defined using the Riesz functional calculus.

Lemma 2.1. If $T$ has $X$ as a spectral set and a normal $B$-dilation, and if $F \in \mathbb{H}(X)$ is unitary valued on $B$, then

$$
\|F(T)\| \leq 1
$$

Proof. Choose a compact set $K$ so that the interior of $K$ contains $X$ and $K$ is a subset of the domain of analyticity of $F$.

Using Runge's Theorem (entrywise), there exists a sequence $G_{n}$ of rational $2 \times 2$ matrix-valued functions with poles off $K$ which converges uniformly to $F$ on $K$. From standard results about the functional calculus, $\left\{G_{n}(T)\right\}$ converges to $F(T)$ in the operator norm. Since $\|F\|_{R}=1$, the sequence $\left\{\left\|G_{n}\right\|_{R}\right\}$ converges to 1 . An application of equation (2.1) and a limit argument completes the proof.

Remark 2.2. The set $X$ is a complete spectral set for an operator $T \in \mathcal{B}(\mathcal{H})$ if $\|F(T)\| \leq\|F\|_{R}$ for every $n \times n$ (no bound on $n$ ) matrix-valued rational function with poles off of $R$ (the norm $\|F\|_{R}$ defined in the expected way). It is a result of Arveson that if $X$ is a complete spectral set for $T$, then $T$ has a normal $B$-dilation [9], 8]; see also [29], 28]. (Arveson's result is actually stated and proved for a commuting $n$-tuple of operators with a domain in $\mathbb{C}^{n}$.)

The following may be found in Conway [18.

Lemma 2.3. If $\pi: \mathbb{H}(X) \rightarrow \mathcal{B}(\mathcal{H})$ is a contractive unital homomorphism, then $X$ is a spectral set for $T=\pi(\zeta)$. Here $\zeta(z)=z$. Moreover, if $f \in \mathbb{H}(X)$, then $\pi(f)=f(T)$.

Proof. Given $\lambda \notin X$, the function $f_{\lambda}(z)=(z-\lambda)^{-1} \in \mathbb{H}(X)$. Thus, as $\pi$ is a unital homomorphism,

$$
I=\pi(1)=\pi\left(f_{\lambda} \cdot(\zeta-\lambda)\right)=\pi\left(f_{\lambda}\right) \pi(\zeta-\lambda)=\pi\left(f_{\lambda}\right)(T-\lambda) .
$$

It follows that the spectrum of $T$ is in $X$.

If $f=p / q \in \mathcal{R}(X), p, q$ polynomials, then $\pi(f)=f(T)$ since $\pi$ is a homomorphism and $f(T)$ can be defined as $p(T) q(T)^{-1}$. Since $\|\pi(f)\| \leq\|f\|_{R}$, it follows that $X$ is a spectral set for $T$.

Finally, if $f \in H(X)$, then by Runge's Theorem, there exists a sequence $\left\{f_{n}\right\}$ from $\mathcal{R}(X)$ which converges uniformly to $f$ on a compact set $K$ containing $X$. By continuity, both $\left\{f_{n}(T)\right\}$ converges to $f(T)$ and $\left\{\pi\left(f_{n}\right)\right\}$ converges to $\pi(f)$ (in the operator norm). Since $f_{n}(T)=\pi\left(f_{n}\right)$, it follows that $f(T)=\pi(f)$.

For $F \in M_{2}(\mathbb{H}(X))$, define

$$
\pi(F)=\left(\pi\left(F_{j, \ell}\right)\right)
$$

Thus, $\pi$ is defined entry-wise.

Proposition 2.4. Suppose $\pi: \mathbb{H}(X) \rightarrow \mathcal{B}(\mathcal{H})$ is a contractive unital homomorphism and let $T=\pi(\zeta)$. If $T$ has a normal $B$-dilation and if $F$ is a $2 \times 2$ matrixvalued function analytic in a neighborhood of $R$ and unitary valued on $B$, then $\|\pi(F)\| \leq 1$. 
Proof. By Lemma 2.1, $\|F(T)\| \leq 1$. On the other hand, by Proposition 2.3, $F(T)=$ $\pi(F)$.

2.2. Harmonic functions. Most of the results and discussion in this section come from Fisher's book 21.

For each point $z \in R$ there exists a measure $\omega_{z}$ on $B$ such that if $h$ is continuous on $X$ and harmonic in $R$, then

$$
h(z)=\int_{B} h(\zeta) d \omega_{z}(\zeta) .
$$

The measure $\omega_{z}$ is a Borel probability measure on $B$ and is known as harmonic measure for the point $z$.

For $z \in R, d \omega_{z}$ and Lebesgue measure $d s$ are mutually absolutely continuous. Thus there is a Radon-Nikodým derivative,

$$
\mathbb{P}(\cdot, z)=\frac{d \omega_{z}}{d s} .
$$

This is also the Poisson kernel for $R$; that is,

$$
\mathbb{P}(\cdot, z)=-\frac{1}{2 \pi} \frac{\partial}{\partial n} g(\cdot, z),
$$

where $g(\cdot, z)$ is the Green's function for the point $z, n$ the outward normal.

If $h$ is a positive harmonic function in $R$, then there exists a positive measure $\mu$ such that

$$
h(z)=\int_{B} \mathbb{P}(\zeta, z) d \mu(\zeta) .
$$

Conversely, given a positive measure $\mu$, the formula (2.2) defines a positive harmonic function in $R$. Note that

$$
h(0)=\int_{B} d \mu=\mu(B) .
$$

Let $h_{j}$ denote harmonic measure for $B_{j}$. Thus, $h_{j}$ is the solution to the Dirichlet problem with boundary values 1 on $B_{j}$ and 0 on $B_{\ell}, \ell \neq j$. Alternatively,

$$
h_{j}(z)=\int_{B_{j}} \mathbb{P}(\zeta, z) d s(\zeta),
$$

where $d s$ is normalized arc length measure for $B$ (Nehari [26, section VII.3).

The symmetry in the domain $R$ yields a simple but useful symmetry for the $h_{j}$.

Lemma 2.5. For $z \in R, h_{j}(z)=h_{j}\left(z^{*}\right)$.

Proof. This is obvious from the definition of $h_{j}(z)$.

2.3. Analytic functions with positive real part. As in the last section, some of the results can be found in the book of Fisher 21. The material on extreme points of the set of normalized analytic functions with positive real part is a special case of that found in Grunsky [22].

If $\mu$ and $h$ are as in the formula (2.2), then the periods $P_{j}(h)$ of the harmonic conjugate of $h$ around $B_{j}, j=0,1,2$, are given by

$$
P_{j}(h)=\int_{B} Q_{j} d \mu
$$

where $Q_{j}$ is the normal derivative of $h_{j}$. Of course $h$ is the real part of an analytic function if and only if $P_{j}(h)=0, j=0,1,2$. 
Lemma 2.6. The functions $Q_{j}$ have no zeros on $B$. Moreover, $Q_{j}>0$ on $B_{j}$ and $Q_{j}<0$ on $B_{\ell}$ for $\ell \neq j$.

Before proving Lemma 2.6 we note the following consequence.

Lemma 2.7. If $h$ is a nonzero positive harmonic function on $R$ which is the real part of an analytic function and if $h$ is represented in terms of a positive measure $\mu$ as in equation (2.2), then $\mu\left(B_{j}\right)>0$ for each $j$.

Proof. If $\mu\left(B_{1}\right)=0$, then, as $Q_{1}<0$ on $B_{0} \cup B_{2}, P_{1}(h)<0$. Thus, $\mu\left(B_{1}\right)>0$. A similar argument holds for $\mu\left(B_{2}\right)$.

Let $h_{0}$ denote harmonic measure for $B_{0}$. Then $\sum_{0}^{2} h_{j}=1$. Consequently, $\sum_{0}^{2} Q_{j}=0$. On the other hand, from the proof of Lemma 2.6. $Q_{0}>0$. Thus, if $\mu\left(B_{0}\right)=0$, then

$$
\begin{aligned}
\sum_{j=1}^{2} \int_{B} Q_{j} d \mu & =\sum_{j, \ell=1}^{2} \int_{B_{\ell}} Q_{j} d \mu \\
& =-\sum_{\ell=1}^{2} \int_{B_{\ell}} Q_{0} d \mu<0,
\end{aligned}
$$

implying that not both $P_{j}(h)=0$. So we must have $\mu\left(B_{0}\right)>0$.

Proof of Lemma 2.6. Let $h_{j}$ denote harmonic measure for $B_{j}$. Thus, $h_{j}$ is the solution to the Dirichlet problem with boundary values 1 on $B_{j}$ and 0 on $B_{\ell}, \ell \neq j$. Alternatively,

$$
h_{j}(z)=\int_{B_{j}} \mathbb{P}(\zeta, z) d s(\zeta),
$$

where $d s$ is normalized arc length measure for $B$.

The functions $Q_{j}$ are related to the $h_{j}$ by

$$
Q_{j}=\frac{\partial h_{j}}{\partial n}
$$

where the derivative is with respect to the outward normal to the boundary. Note that, for $j=0,1,2$, the partial derivatives $\frac{\partial h_{j}}{\partial n}$ are evidently nonnegative on $B_{j}$ and nonpositive on $B_{\ell}$, for $\ell \neq j$, from which it follows that $Q_{j} \geq 0$ on $B_{j}$ and $Q_{j} \leq 0$ on $B_{\ell}$ for $\ell \neq j$.

Let $R^{\prime}$ denote the reflection of $R$ about $B_{0}$ by $z \mapsto 1 / z^{*}$. The functions $h_{j}$ naturally extend to harmonic functions on $X \cup R^{\prime}$ by

$$
h_{j}\left(1 / z^{*}\right)=-h_{j}(z) \text {. }
$$

Thus, $Q_{j}$ extends to $R^{\prime}$.

If $Q_{j}$ were to have infinitely many zeros in $B$, then $Q_{j}$ would be zero. By the Cauchy-Riemann equations, $\tilde{h}_{j}$, a harmonic conjugate of $h_{j}$ exists on $B_{0}$ for $j=1,2$. Moreover, since $Q_{j} \leq 0$ on $B_{0}$ and has only finitely many zeros on $B_{0}, \tilde{h}_{j}$ is strictly decreasing (in the positive orientation on $B_{0}$ ). In particular, $\tilde{h}_{j}$ is one-one on $B_{0}$. Observe, if $Q_{j}$ is zero at a point $\zeta \in B_{0}$, then, as the derivative of $h_{j}$ tangential to $B$ at $\zeta$ is also 0 , the function $f=h_{j}+i \tilde{h_{j}}$ is analytic near $\zeta$ and has zero derivative at $\zeta$. It follows that $f$ is at least two 
to one in a sufficiently small neighborhoods of $\zeta$. But then $\tilde{h_{j}}$ could not be one to one on $B_{0}$ near $\zeta$, a contradiction. Hence $Q_{j}<0$ on $B_{0}(j=1,2)$.

Similar arguments show $Q_{j}>0$ on $B_{j}$ and $Q_{j}<0$ on $B_{\ell}$ for $\ell \neq j$.

Let $\Pi=B_{0} \times B_{1} \times B_{2}$.

Lemma 2.8. For each $p \in \Pi$, the kernel of

$$
M(p)=\left(\begin{array}{lll}
Q_{1}\left(p_{0}\right) & Q_{1}\left(p_{1}\right) & Q_{1}\left(p_{2}\right) \\
Q_{2}\left(p_{0}\right) & Q_{2}\left(p_{1}\right) & Q_{2}\left(p_{2}\right)
\end{array}\right)
$$

is one-dimensional and spanned by a vector with all entries strictly positive. In particular, there is a continuous function $\tau: \Pi \rightarrow \mathbb{R}^{3}$ such that $\tau(p)$ is entry-wise positive, the sum of the entries is one, and $\tau(p)$ is in the kernel of $M(p)$.

Moreover, $\tau$ reflects the symmetry in the domain. Namely,

$$
\tau\left(p_{0}, p_{1}, p_{2}\right)=\tau\left(p_{0}^{*}, p_{1}, p_{2}\right)=\tau\left(p_{0}, p_{1}^{*}, p_{2}\right)=\tau\left(p_{0}, p_{1}, p_{2}^{*}\right) .
$$

Proof. Computing the cross product of the rows of $M(p)$, we get the vector with entries

$$
\begin{aligned}
& Q_{1}\left(p_{1}\right) Q_{2}\left(p_{2}\right)-Q_{1}\left(p_{2}\right) Q_{2}\left(p_{1}\right), \\
& Q_{1}\left(p_{2}\right) Q_{2}\left(p_{0}\right)-Q_{1}\left(p_{0}\right) Q_{2}\left(p_{2}\right), \\
& Q_{1}\left(p_{0}\right) Q_{2}\left(p_{1}\right)-Q_{1}\left(p_{1}\right) Q_{2}\left(p_{0}\right) .
\end{aligned}
$$

This vector is in the kernel of $M(p)$ and by considering the signs of the $Q_{j}$ on the boundary components $B_{\ell}$, one easily checks that the signs of the last two entries are positive. It is also clear from the signs of the entries that the rows of $M(p)$ are linearly independent. Hence $M(p)$ is rank two and its kernel is one dimensional. To finish the proof of the first part of the lemma, it remains to show that the sign of the first entry is positive. Since $\sum_{j=0}^{2} h_{j}(P)=1$ for $P \in X$, it follows that for $P \in B_{1}$ or $P \in B_{2}$, that

$$
Q_{1}(P)+Q_{2}(P)=-Q_{0}(P)>0 .
$$

Thus,

$$
\begin{aligned}
Q_{1}\left(p_{1}\right) Q_{2}\left(p_{2}\right) & -Q_{1}\left(p_{2}\right) Q_{2}\left(p_{1}\right) \\
& =Q_{1}\left(p_{1}\right) Q_{2}\left(p_{2}\right)+Q_{1}\left(p_{1}\right) Q_{1}\left(p_{2}\right)-Q_{1}\left(p_{1}\right) Q_{1}\left(p_{2}\right)-Q_{1}\left(p_{2}\right) Q_{2}\left(p_{1}\right) \\
& =Q_{1}\left(p_{1}\right)\left(Q_{2}\left(p_{2}\right)+Q_{1}\left(p_{2}\right)\right)-Q_{1}\left(p_{2}\right)\left(Q_{1}\left(p_{1}\right)+Q_{2}\left(p_{1}\right)\right) \\
& =-Q_{1}\left(p_{1}\right) Q_{0}\left(p_{2}\right)+Q_{1}\left(p_{2}\right) Q_{0}\left(p_{1}\right) .
\end{aligned}
$$

Examination of the signs of the terms on the right-hand side above shows that the first term is indeed positive.

To prove the last part of the lemma, simply note that the symmetry in the domain implies each $h_{j}$ is symmetric, $h_{j}\left(z^{*}\right)=h_{j}(z)$ and thus, $Q_{j}\left(z^{*}\right)=Q_{j}(z)$.

The lemma allows the construction of canonical analytic functions of positive real part on $R$, since, by construction,

$$
h_{p}=\sum \tau_{j}(p) \mathbb{P}\left(\cdot, p_{j}\right)
$$

is a positive harmonic function with no periods. Indeed, $h_{p}$ corresponds to the measure $\sum \tau_{j}(p) \delta_{p_{j}}$ in equation (2.2). In particular, by our normalization of $\tau$,

$$
h_{p}(0)=1 .
$$

Let $f_{p}$ denote the analytic function with real part $h_{p}$ such that $f(p)=1$. 
Let $H(R)$ be the locally convex metrizable topological space of holomorphic functions on $R$ with the topology of uniform convergence on compact subsets of $R$. The space $H(R)$ has the Heine-Borel property; i.e., closed bounded subsets of $H(R)$ are compact. Let

$$
\mathbb{K}=\left\{f \in H(R): f(0)=1, f+f^{*}>0\right\} .
$$

The set $\mathbb{K}$ is easily seen to be closed.

Lemma 2.9. The set $\mathbb{K}$ is compact.

What needs to be shown is that $\mathbb{K}$ is bounded-in other words, that for each compact subset $K \subset R$ there exists an $M_{K}$ so that $|f(z)| \leq M_{K}$ for all $f \in \mathbb{K}$ and $z \in K$.

That $\mathbb{K}$ is bounded is straightforward in the case that $R$ is replaced by the unit disk, and we consider this case to begin with.

Let $P_{r}(\theta)$ denote the Poisson kernel and $Q_{r}(\theta)$ the conjugate Poisson kernel,

$$
\begin{aligned}
P_{r}(\theta) & =\frac{1-r^{2}}{1+r^{2}-2 r \cos (\theta)} \\
Q_{r}(\theta) & =\frac{2 r \sin (\theta)}{1+r^{2}-2 r \cos (\theta)} .
\end{aligned}
$$

If $U$ is a positive harmonic in the unit disk $\mathbb{D}$ with $U(0)=1$, then there is a probability measure $\mu$ so that

$$
U(r \exp (i \theta))=\int_{-\pi}^{\pi} P_{r}(\theta-t) d \mu(t) .
$$

The harmonic conjugate $V$ of $U$ normalized by $V(0)=0$ is given by

$$
V(r \exp (i \theta))=\int_{-\pi}^{\pi} Q_{r}(\theta-t) d \mu(t) .
$$

Thus, as $\mu$ is a probability measure, for each $0<r<1$ there is a constant $M_{r}$ so that if $|z| \leq r$, then $|U(z)|,|V(z)| \leq M_{r} / 2$. By conformal mapping, the result is seen to hold for any bounded simply connected domain.

The proof of the lemma is based upon the above result for simply connected domains, using the fact that $R$ can be written as the union of two simply connected domains.

Proof of Lemma 2.9. It suffices to show that for each compact set $K$ there is a constant $M_{K}$ so that if $f \in \mathbb{K}$ and $z \in K,|f(z)| \leq M_{K}$. In fact, it is enough to work with the compact sets of the form

$$
K_{\epsilon}=\{|z| \leq 1-\epsilon\} \cap\left\{\left|z+c_{1}\right| \geq r_{1}+\epsilon\right\} \cap\left\{\left|z+c_{2}\right| \geq r_{2}+\epsilon\right\},
$$

for $\epsilon>0$ sufficiently small.

First, observe that if $f$ is holomorphic with positive real part on $R$ and $f(0)=1$, then for each $\epsilon>0$ there is a constant $N_{\epsilon}$ so that for all $z \in K_{\epsilon}, h(z) \leq N_{\epsilon}$, by virtue of the representation (2.2).

Given $\epsilon>0$, the open simply connected sets

$$
R_{\epsilon}^{ \pm}=\left\{z=a+b i \in K_{\frac{\epsilon}{2}}: \pm b \geq-\frac{\min \left(r_{1}, r_{2}\right)}{2}\right\}
$$


contain 0 and their union is $K_{\frac{\epsilon}{2}}$. After conformal mapping from the disk taking 0 to 0 , it follows that there exists $M_{\epsilon}^{ \pm}$so that if $f$ is analytic on $R$ with $f(0)=1$, then for all $z \in R_{\epsilon}^{ \pm},|f(z)| \leq M_{\epsilon}^{ \pm}$.

Lemma 2.10 ([22]). The extreme points of $\mathbb{K}$ are precisely $\left\{f_{p}: p \in \Pi\right\}$.

Proof. It is evident that each $f_{p}$ is an extreme point of $\mathbb{K}$. So consider the converse.

Let $f \in \mathbb{K}$. The real part of $f$ is a positive harmonic function $h$ with $h(0)=1$. Hence there exists a probability measure $\mu$ such that

$$
h(z)=\int_{B} \mathbb{P}(\zeta, z) d \mu(\zeta) .
$$

Suppose the support of $\mu$ on $B_{0}$ contains more than one point and so can be written as the union of disjoint sets $A_{1}, A_{2} \subset B_{0}$ with $\mu\left(A_{j}\right)>0$.

Let

$$
\alpha_{j, \ell}=\int_{A_{\ell}} Q_{j} d \mu, \quad \ell=1,2
$$

and

$$
\kappa_{j, m}=\int_{B_{m}} Q_{j} d \mu, \quad m=1,2 .
$$

Since $h$ is the real part of an analytic function,

$$
0=\int_{B} Q_{j} d \mu
$$

Thus, $\kappa_{j, 1}+\kappa_{j, 2}+\left(\alpha_{j, 1}+\alpha_{j, 2}\right)=0$. Since also $Q_{j}<0$ on $B_{0}$ for $j=1,2$,

$$
\kappa_{j, 1}+\kappa_{j, 2}=-\left(\alpha_{j, 1}+\alpha_{j, 2}\right)>0 .
$$

This gives $\kappa_{1,1} \geq\left|\kappa_{1,2}\right|=-\kappa_{1,2}$. Hence the determinant of $\kappa=\left(\kappa_{i, j}\right)$ is positive.

Since the determinant of $\kappa=\left(\kappa_{i, j}\right)$ is positive the solution of

$$
\left(\begin{array}{ll}
\kappa_{1,1} & \kappa_{1,2} \\
\kappa_{2,1} & \kappa_{2,2}
\end{array}\right)\left(\begin{array}{l}
\beta_{1, \ell} \\
\beta_{2, \ell}
\end{array}\right)=-\left(\begin{array}{l}
\alpha_{1, \ell} \\
\alpha_{2, \ell}
\end{array}\right)
$$

is given by

$$
\frac{1}{\operatorname{det} k}\left(\begin{array}{cc}
\kappa_{2,2} & -\kappa_{1,2} \\
-\kappa_{2,1} & \kappa_{1,1}
\end{array}\right)\left(\begin{array}{l}
-\alpha_{1, \ell} \\
-\alpha_{2, \ell}
\end{array}\right)=\left(\begin{array}{c}
\beta_{1, \ell} \\
\beta_{2, \ell}
\end{array}\right) .
$$

In view of the signs of the $\kappa_{j, m}$ and $\alpha_{j, \ell}<0$, it follows that $\beta_{1, \ell}, \beta_{2, \ell} \geq 0$.

Define positive measures $\nu_{\ell}$ by

$$
\nu_{\ell}(A)=\mu\left(A \cap A_{\ell}\right)+\beta_{1, \ell} \mu\left(A \cap B_{1}\right)+\beta_{2, \ell} \mu\left(A \cap B_{2}\right) .
$$

Then

$$
\int_{B} Q_{j} d \nu_{\ell}=\alpha_{j, \ell}+\kappa_{j, 1} \beta_{1, \ell}+\kappa_{j, 2} \beta_{2, \ell}=0
$$

and therefore each

$$
h_{\ell}=\int_{B} \mathbb{P}(\cdot, \zeta) d \nu_{\ell}(\zeta)
$$

is the real part of an analytic function $g_{\ell}$ with $g_{\ell}(0)=\nu(B)$. Since also $\nu_{1}+\nu_{2}=\mu$, we have $h_{1}+h_{2}=h$. Thus, $\frac{g_{\ell}}{g_{\ell}(0)} \in \mathbb{K}$ and

$$
f=g_{1}(0)\left(\frac{g_{1}}{g_{1}(0)}\right)+g_{2}(0)\left(\frac{g_{2}}{g_{2}(0)}\right) \text {. }
$$

We conclude that $f$ is not an extreme point. 
Next suppose $B_{1}$ is a disjoint union of sets $A_{1}, A_{2}$ with $\mu\left(A_{j}\right)>0$. Let

$$
\alpha_{j, \ell}=\int_{A_{\ell}} Q_{j} d \mu, \quad \ell=1,2
$$

and

$$
\kappa_{j, m}=\int_{B_{m}} Q_{j} d \mu, \quad m=0,1 .
$$

This time the signs are $\alpha_{1, \ell} \geq 0, \alpha_{2, \ell} \leq 0, \kappa_{j, 0} \leq 0, \kappa_{1,2}<0$, and $\kappa_{2,2}>0$. So, with

$$
\kappa=\left(\begin{array}{ll}
\kappa_{1,0} & \kappa_{1,2} \\
\kappa_{2,0} & \kappa_{2,2}
\end{array}\right)
$$

the determinant of $\kappa$ is negative. Thus, the solution of

$$
\kappa\left(\begin{array}{c}
\beta_{0, \ell} \\
\beta_{2, \ell}
\end{array}\right)=-\left(\begin{array}{c}
\alpha_{1, \ell} \\
\alpha_{2, \ell}
\end{array}\right)
$$

is given by

$$
\frac{1}{\operatorname{det} k}\left(\begin{array}{cc}
\kappa_{2,2} & -\kappa_{1,2} \\
-\kappa_{2,0} & \kappa_{1,0}
\end{array}\right)\left(\begin{array}{l}
-\alpha_{1, \ell} \\
-\alpha_{2, \ell}
\end{array}\right)=\left(\begin{array}{c}
\beta_{1, \ell} \\
\beta_{2, \ell}
\end{array}\right) .
$$

In view of the signs of the entries and of $\operatorname{det}(\kappa)$, the $\beta_{j, \ell}$ are all nonnegative.

Define positive measures $\nu_{\ell}$ by

$$
\nu_{\ell}(A)=\mu\left(A \cap A_{\ell}\right)+\beta_{0, \ell} \mu\left(A \cap B_{0}\right)+\beta_{2, \ell} \mu\left(A \cap B_{2}\right) .
$$

Then

and therefore each

$$
\int_{B} Q_{j} d \nu_{\ell}=\alpha_{j, \ell}+\kappa_{j, 0} \beta_{0, \ell}+\kappa_{j, 2} \beta_{2, \ell}=0
$$

$$
h_{\ell}=\int_{B} \mathbb{P}(\cdot, \zeta) d \nu_{\ell}(\zeta)
$$

is the real part of an analytic function $g_{\ell}$ with $g_{\ell}(0)=\nu(B)$. Since also $\nu_{1}+\nu_{2}=\mu$, we have $h_{1}+h_{2}=h$. The argument proceeds as before, with $\frac{g_{\ell}}{g_{\ell}(0)} \in \mathbb{K}$ and

$$
f=g_{1}(0)\left(\frac{g_{1}}{g_{1}(0)}\right)+g_{2}(0)\left(\frac{g_{2}}{g_{2}(0)}\right) \text {. }
$$

We conclude that $f$ is not an extreme point.

Lemma 2.11. The set of extreme points of $\mathbb{K}$ is a closed set and the function taking $\Pi$ to $\mathbb{K}$ by $p \mapsto f_{p}$ is a homeomorphism.

Proof. It suffices to show, if $p(n) \in \Pi$ converges to $p(0)$ in $\Pi$, then $f_{p(n)}$ converges uniformly on compact subsets of $R$ to $f_{p(0)}$. Since $\mathbb{K}$ is compact and $\left\{f_{p(n)}\right\}$ is a sequence in $\mathbb{K}$, some subsequence, still denoted $\left\{f_{p(n)}\right\}$, converges to some $f \in$ $\mathbb{K}$. Let $h_{p(n)}$ denote the real part of $f_{p(n)}$ and let $\mu_{n}$ denote the measure which represents $h_{p(n)}$ so that

$$
h_{p(n)}(z)=\int_{B} \mathbb{P}(\zeta, z) d \mu_{n}
$$

and $\mu_{n}=\sum \tau_{j}(p(n)) \delta_{p(n)_{j}}$, where $\tau$ is defined in Lemma 2.8. See also equation (2.3).

The measures $\mu_{n}$ converge to the measure $\mu_{0}$ weakly so that $h_{p(n)}(z)$ converges to $h_{p(0)}(z)$ pointwise in $R$. It follows that $h_{p(0)}$ is the real part of $f$. Thus, $\left\{f_{p(n)}\right\}$ converges, in $H(R)$, to $f_{p(0)}$. Since every subsequence of our original subsequence has a subsequence which converges to $f_{p(0)}$, the whole subsequence converges to 
$f_{p(0)}$. This shows that the mapping is continuous. Since $\Pi$ is compact, it follows that our mapping is a homeomorphism and its range is compact.

2.4. Scalar inner functions on $R$. Up to post composition by a Möbius transformation, the inner functions on $R$ with precisely three zeros are canonically parameterized by the 2 -torus, $\mathbb{T}^{2}$. Details may be found in the book by Fay [20]. This section contains an alternate description of this family using results from the previous sections. The following is well known (see, for example, Fisher [21], Ch. 4, ex. 6,7$)$.

Proposition 2.12. A nonconstant inner function $\psi$ on $R$ has at least three zeros counting with multiplicity. Moreover, if $\psi$ has exactly three zeros, $z_{0}, z_{1}, z_{2}$, then for $j=1,2$,

$$
\sum_{\ell=0}^{2} h_{j}\left(z_{\ell}\right)=1 .
$$

As a first application, the last proposition allows us to show that we may assume that our region supports no inner function with a zero of multiplicity three at 0 , and so consequently we will take it that $R$ has this property throughout the remainder of the paper.

Corollary 2.13. We may assume without loss of generality that there is no inner function on $R$ with a zero of multiplicity three at 0 .

Proof. Suppose $f$ is an inner function on $R$ with exactly three zeros counting multiplicity. Then if $f$ takes the value $f(x)$ with multiplicity three where $x \in$ $\left(c_{1}+r_{1}, c_{2}-r_{2}\right)$ (i.e., $f(z)-f(x)$ has a zero of multiplicity three at $x$ ), there is a Möbius transformation $m$ of the disk to itself fixing 1 and moving $f(x)$ to 0 . Thus $m \circ f$ is an inner function with zero of multiplicity three at the origin. Hence by Proposition 2.12 $h_{j}(x)=1 / 3$. Since $h_{1}$ is continuous and equal to 1 on $B_{1}$, if we choose $x$ close enough to $c_{1}+r_{1} \in B_{1}$, it will be the case that $h_{1}(x)>1 / 3$, and so $f$ does not take the value $f(x)$ with multiplicity three for any inner function with three zeros. Now take a Möbius transformation mapping $x$ to the origin. This takes $R$ to a new region which again has the property that it is a region which is the unit disk with two smaller disks removed on either side of the imaginary axis and centers on the real axis.

For $p \in \Pi$, let

$$
\phi_{p}=\frac{f_{p}-1}{f_{p}+1}
$$

where $f_{p}$ is the extreme point of $\mathbb{K}$ corresponding to the point $p=\left(p_{0}, p_{1}, p_{2}\right)$ in $\Pi$ as in the previous subsection. The real part, $h_{p}$, of $f_{p}$ is harmonic across $B \backslash\left\{p_{0}, p_{1}, p_{2}\right\}$ and therefore $f_{p}$ is (at least locally) analytic across $B \backslash\left\{p_{0}, p_{1}, p_{2}\right\}$. Furthermore, near $p_{j}, f_{p}$ has the form $\frac{g_{j}(z)}{z-p_{j}}$ for some $g_{j}$ analytic in a neighborhood of $s_{j}$ and nonvanishing at $p_{j}\left(21\right.$, Ch. 4). From these properties of $f_{p}$, it follows that $\phi_{p}$ is continuous onto $B$ and $\left|\phi_{p}\right|=1$ on $B$. By the reflection principle, $\phi_{p}$ extends to be analytic in a neighborhood of $B$. Since $\phi_{p}$ is inner and extends analytically across $B$, and $\phi_{p}^{-1}(\{1\})=\left\{p_{0}, p_{1}, p_{2}\right\}$, it follows that the preimage of each point $z \in \mathbb{D}$ is exactly three points, counted with multiplicity. In particular, $\phi_{p}$ has precisely three zeros. 
On the other hand, suppose $\phi$ is analytic in a neighborhood of $R$, has modulus one on $B$, and three zeros in $R$. As above, it follows that $\phi^{-1}(1)$ consists of three points. Moreover, the real part of

$$
f=\frac{1+\phi}{1-\phi}
$$

is a positive harmonic function which is zero on $B$ except at those points $z$ where $\phi(z)=1$. By Lemma 2.7 we must have $\phi^{-1}(\{1\})=\left\{p_{0}, p_{1}, p_{2}\right\}$ where $p_{j} \in B_{j}$. So if we also assume $\phi(0)=0$, then $\phi=\phi_{p}$ for some $p \in \Pi$.

Proposition 2.14. If $\psi$ is analytic in $R$ and if $|\psi| \leq 1$ on $R$, then there exists a positive measure $\mu$ on $\Pi$ and a measurable function $\bar{h}$ defined on $\Pi$ whose values are functions $h(\cdot, p)$ analytic in $R$ so that

$$
1-\psi(z) \psi(w)^{*}=\int h(z, p)\left[1-\phi_{p}(z) \phi_{p}(w)^{*}\right] h(w, p)^{*} d \mu(p) .
$$

Proof. First suppose $\psi(0)=0$.

Let

$$
f=\frac{1+\psi}{1-\psi}
$$

Verify

$$
\psi=\frac{f-1}{f+1}
$$

and hence,

$$
1-\psi(z) \psi(w)^{*}=2 \frac{f(z)+f(w)^{*}}{(f(z)+1)\left(f(w)^{*}+1\right)} .
$$

Since $h$, the real part of $f$, is positive and $f(0)=1$, the function $f$ is in $\mathbb{K}$ defined in the previous subsection.

Since $\mathbb{K}$ is a compact subset of the topological vector space $H(R)$ and the set of extreme points $\left\{f_{p}: p \in \Pi\right\}$ of $\mathbb{K}$ is a compact set by Lemma 2.11] there exists a (regular Borel) probability measure $\nu$ on $\Pi$ so that

$$
f=\int_{\Pi} f_{p} d \nu(p)
$$

Using the definition of $\phi_{p}$ and equation (2.4), verify

$$
1-\psi(z) \psi(w)^{*}=\int_{\Pi} \frac{1-\phi_{p}(z) \phi_{p}(w)^{*}}{(f(z)+1)\left(1-\phi_{p}(z)\right)\left(1-\phi_{p}(w)^{*}\right)\left(f(w)^{*}+1\right)} d \nu(p) .
$$

If $\psi(0)=a$, then one has a representation as above since

$$
1-\left(\frac{\psi(z)-a}{1-a^{*} \psi(z)}\right)\left(\frac{\psi(w)-a}{1-a^{*} \psi(w)}\right)^{*}=\frac{\left(1-a a^{*}\right)\left(1-\psi(z) \psi(w)^{*}\right)}{\left(1-a^{*} \psi(z)\right)\left(1-a \psi(w)^{*}\right)} .
$$

While we have used the three parameters in $\Pi$ to parameterize the inner functions with exactly three zeros, after rotation, really only two are needed. Indeed,

$$
\phi_{p}(1)^{*} \phi_{p}=\phi_{q}
$$

where $q=\left(1, q_{1}, q_{2}\right), q_{j} \in B_{j}$ for $j=1,2$, are the unique points such that $\phi_{p}\left(q_{j}\right)=$ $\phi_{p}(1)$. 


\section{Some Riemann SURfaces And theta FunCtions}

We review some of the results from the theory of Riemann surfaces which we will need subsequently. In particular, we look at theta functions, the use of which in operator theory was pioneered by Clancey [15], 16]. The presentation here borrows heavily from Ball and Clancey [10] as well as Mumford [24, 25], and Farkas and Kra 19.

Let $Y$ denote the double of the bordered Riemann surface $X=R \cup B$. Recall $Y$ is obtained topologically by gluing a second copy $R^{\prime}$ of $R$ along $B$. The complex atlas is then found by anti-holomorphically reflecting the complex structure from $X$ to $R^{\prime}$. Since $B_{0}$ is the unit circle, the anti-copy $R^{\prime}$ of $R$ may be thought of as the reflection of $R$ in $B_{0}$ given by $z \mapsto z^{*-1}$. In particular, there is an anti-holomorphic involution $J: Y \rightarrow Y$ which fixes $B$. For $\zeta \in R, J \zeta$ is its twin in $R^{\prime}$.

Note that $Y$ is a compact Riemann surface. It is important since we can sometimes extend analytic functions defined on $R$ to meromorphic functions on $Y$. In particular this is true for inner functions, which extend by reflection.

We get a lot of mileage out of a few basic tools for analyzing meromorphic functions on compact Riemann surfaces. To begin with, if such a function is nonconstant and takes a given value $n$ times, it takes all of its values $n$ times (counting multiplicity). Furthermore, and as a consequence, such a function has equal numbers of zeros and poles. Finally, a meromorphic function on a compact Riemann surface without zeros or poles is constant.

The material in subsections 3.3 3.5 is solely for describing the zeros of the Fay kernel in Section 4 . The reader who is willing to accept the statement of Theorem 4.2 may skip these parts.

3.1. Minimal meromorphic functions on $R$. Our Riemann surface $Y$ also comes naturally equipped with a conformal involution which fixes 6 points, namely

$$
\iota(\zeta)=J\left(\zeta^{*}\right)
$$

Geometrically, $\iota$ is rotation by $\pi$ in the axis through \pm 1 . The fixed points of the involution are precisely the Weierstrass points of $Y$, namely $\pm 1, c_{1} \pm r_{1}, c_{2} \pm r_{2}$ ([19], Cor. 1, p. 108).

If $f$ is a meromorphic function on a compact Riemann surface, then there is a number $n$, the degree of $f$, so that $f$ takes each value $n$ times, counting multiplicity. In particular, if $f$ has just one pole, then $f$ is one to one and the Riemann surface is conformally equivalent to the Riemann sphere. Thus, a nonconstant meromorphic function on $Y$ must have at least two poles (and zeros).

Up to post composition by a Möbius transformation, there is a unique meromorphic function $\phi$ on $Y$ with precisely two poles. Moreover, $\phi$ is ramified with branching one at the Weierstrass points.

The construction of a meromorphic function with two poles and zeros found in Farkas and Kra ([19], Theorem III.7.3) is the following. The Riemann surface $\tilde{Y}$, obtained as the quotient of $Y$ by the map $\zeta \mapsto \iota^{-1}(\zeta)$, is a Riemann surface of genus zero by the Riemann-Hurwitz formula. Thus, $\tilde{Y}$ is conformally equivalent to the Riemann sphere, and the canonical quotient map

$$
Y \mapsto \tilde{Y}
$$


followed by a conformal map from $\tilde{Y}$ to the Riemann sphere generates the desired meromorphic function. From this construction it is evident that if $\phi$ is any meromorphic function with two poles and two zeros, then $\phi \iota=\phi$. Another way to see this is that $\phi-\phi \iota$ has zeros at the six Weierstrass points, while it has at most four poles, and so must be constantly zero.

Proposition 3.1. If $f$ is a meromorphic function on $Y$ with precisely two poles $Q_{1}$ and $Q_{2}$, then $Q_{2}=\iota\left(Q_{1}\right)$. In particular, $f$ cannot have one pole in $R$ and the other in $X=R \cup B$. The same statement holds if we consider zeros rather than poles.

Proof. From the discussion above, $f \circ \iota=f$. The conclusion for the poles of $f$ follows. By considering $1 / f$ the conclusion for the zeros of $f$ is also seen to hold.

Using the proposition it is possible to show that a unimodular function on $R$ which takes the value 1 at exactly one point on each boundary component $B_{j}$ and is 0 at 0 is uniquely determined. Of course, this is also evident by construction and has already been used.

Corollary 3.2. Suppose $p_{j} \in B_{j}$, for $j=0,1,2$. If $f, g: X \rightarrow \mathbb{C}$ are analytic in a neighborhood of $X$, unimodular on $B, f^{-1}(\{1\}) \cap X=g^{-1}(\{1\}) \cap X=\left\{p_{0}, p_{1}, p_{2}\right\}$, and $f(0)=g(0)=0$, then $f=g$.

Proof. By the maximum modulus principle, $f$ and $g$ are strictly less than one in modulus in $R$. The functions $f$ and $g$ reflect to meromorphic functions on $Y$, which can take the value 1 only on the boundary of $B$. Thus, the functions are globally three to one and so have three zeros in $R$ and three poles, at the reflected points, in $R^{\prime}$. In particular, $f$ and $g$ share the zero 0 and pole $J 0$.

Consider the function

$$
\psi=\frac{1-f}{1-g} .
$$

The zeros of $1-f$ and those of $1-g$ agree, so that $\psi$ can only have poles at the poles of $f$. Since one of these poles matches a pole of $g, \psi$ has only two poles (and two zeros). However, these poles are poles of $f$ which both are in $R^{\prime}$, contradicting the proposition. Hence, it must be that $\psi$ is constant so that $1-f=c(1-g)$. Since $f(0)=g(0)=0, c=1$.

We can also describe minimal inner functions which agree at two of their zeros.

Lemma 3.3. If $\phi_{p}$ and $\phi_{\tilde{p}}$ have two zeros in common (0 and one other) and $\phi_{p}(1)=$ $\tilde{\phi}_{p}(1)=1$, then either they are equal or the remaining zeros form a conjugate pair. In the case that $p=\left(1, p_{1}, p_{2}\right)$ and $\tilde{p}=\left(1, p_{1}, \tilde{p}_{2}\right)$, then $\tilde{p}_{2}=p_{2}$ and so $\phi_{\tilde{p}}=\phi_{p}$.

Proof. If $\phi_{p}$ and $\phi_{\tilde{p}}$ have all three zeros in common, then they are equal, and there is nothing to prove. So assume instead that they have two common zeros $\left(0\right.$ and $\left.z_{1}\right)$ and unequal zeros $z_{2}, \tilde{z}_{2}$ for $\phi_{p}$ and $\phi_{\tilde{p}}$, respectively. Then $\phi_{p} / \phi_{\tilde{p}}$ has two simple zeros and poles, $z_{2}, J \tilde{z}_{2}$ and $\tilde{z}_{2}, J z_{2}$, respectively. By Proposition 3.1. $J z_{2}=\iota \tilde{z}_{2}=J\left(\tilde{z}_{2}^{*}\right)$, or $\tilde{z}_{2}=z_{2}^{*}$.

Now suppose that $\phi_{p}$ and $\phi_{\tilde{p}}$ have common zeros 0 and $z_{1}$, and that $p=\left(1, p_{1}, p_{2}\right)$ and $\tilde{p}=\left(1, p_{1}, \tilde{p}_{2}\right)$. Then unless $\phi_{p} / \phi_{\tilde{p}}$ is constant, $\phi_{p} / \phi_{\tilde{p}}-1$ has the same two poles as $\phi_{p} / \phi_{\tilde{p}}$. It would then have exactly two zeros, namely 1 and $p_{1}$. Now apply Proposition 3.1 and note that $\iota(1)=1 \neq p_{1}$. The result then follows. 
Lemma 3.4. Let $p=\left(1, p_{1}, p_{2}\right)$. If the zeros of $\phi_{p}$ are all real, then $p_{1}, p_{2} \in \mathbb{R}$.

Conversely, for $p=\left(1, c_{1}-r_{1}, c_{2}-r_{2}\right)$, the zeros of $\phi_{p}$ are all real. In fact, $\phi_{p}$ has a zero at zero and one zero in each of the intervals $\left(-1, c_{1}-r_{1}\right)$ and $\left(c_{2}+r_{2}, 1\right)$ on the real axis in $R$.

It turns out that for $p=\left(1, c_{1}+r_{1}, c_{2}-r_{2}\right)$ or $p=\left(1, c_{1}-r_{1}, c_{2}+r_{2}\right)$, the zeros of $\phi_{p}$ are also real.

Proof. Suppose that the zeros of $\phi_{p}$ are real, and define $\tilde{\phi}_{p}=\phi_{p}\left(\zeta^{*}\right)^{*}$. Then $\tilde{\phi}_{p}$ has the same zeros as $\phi_{p}$. Hence the ratio of these two functions has no zeros and so must be constant. Since both equal 1 at 1 , it follows that they are equal everywhere. In particular,

$$
\phi_{p}\left(c_{j} \pm r_{j}\right)^{*}=\phi_{p}\left(\left(c_{j} \pm r_{j}\right)^{*}\right)^{*}=\phi_{p}\left(c_{j} \pm r_{j}\right)
$$

so that $\phi_{p}\left(c_{j} \pm r_{j}\right)$ is real (in each of the four cases). Since $\phi_{p}$ maps each $B_{j}$ one-one onto the unit circle, it follows that $\phi_{p}$ takes the value 1 at one of the points $c_{j} \pm r_{j}$ on $B_{j}, j=1,2$ and this gives the conclusion of the lemma.

For the converse, define $\tilde{\phi}_{p}$ as above. Since the entries of $p$ are real, Corollary 3.2 implies $\phi_{p}(\zeta)=\tilde{\phi}_{p}\left(\zeta^{*}\right)^{*}$ so that $\phi_{p}$ is real on the real axis. Since also $\phi_{p}$ takes the value 1 at $c_{j}-r_{j}$ and by assumption there is only one point on $B_{j}$ where $\phi_{p}$ equals 1 , it takes the value -1 at the points $c_{j}+r_{j}$. The intermediate value theorem now implies that $\phi_{p}$ has at least one zero in each of the intervals, $\left(-1, c_{1}-r_{1}\right)$, $\left(c_{1}+r_{1}, c_{2}-r_{2}\right)$, and $\left(c_{2}+r_{2}, 1\right)$. Since $\phi_{p}$ has exactly three zeros the result follows.

For $t=\left(t_{1}, t_{2}\right), t_{1}, t_{2} \in[0,2 \pi)$, let $p_{1}(t)=c_{1}-r_{1} \exp \left(i t_{1}\right)$ and $p_{2}=c_{2}-$ $r_{2} \exp \left(i t_{2}\right)$. These points are on the two inner components of the boundary. On the outer boundary we just use 1 . Accordingly, let $p(t)=\left(1, p_{1}(t), p_{2}(t)\right) \in \Pi$ and, for notational ease, let $\psi_{t}=\phi_{p(t)}$. Then $\psi_{-t}=\phi_{p(-t)}$.

Lemma 3.5. There exist $t_{1}, t_{2}$ nonzero such that $\psi_{t}$ has three distinct zeros $0, z_{1}, z_{2}$ with $z_{1}, z_{2}$ both nonreal.

Proof. Write 0 for $(0,0)$. From the second part of Lemma 3.4, $\psi_{0}$ has distinct real zeros.

Now suppose that $t=\left(t_{1}, 0\right)$. If $\pi>t_{1}>0$ is small enough, the zeros $0, z_{1}, z_{2}$ of $\psi_{t}$ are still distinct. By the first part of Lemma 3.4, if these zeros are all real, then $t_{1}$ is a multiple of $\pi$. On the other hand suppose that one of the nonzero zeros of $\psi_{t}$, say $z_{1}$, is real. Then $\psi_{t}$ and $\psi_{-t}$ share 0 and $z_{1}$ as zeros, as well as both being equal to 1 at 1 and $c_{2}-r_{2}$. Hence by Lemma 3.3 they are equal, implying that $p_{1}(t)$ is real, or equivalently, that $t_{1}$ is a multiple of $\pi$. Since we have chosen $0<t_{1}<\pi$, this cannot happen. Thus we must have that both zeros of $\psi_{t}$ are nonreal for small enough $t_{1}$.

Finally, if we choose $t_{1}$ such that $\psi_{\left(t_{1}, 0\right)}$ has nonreal roots, then by continuity, for small enough $t_{2}, \psi_{t}$ will still have nonreal roots with $t=\left(t_{1}, t_{2}\right)$. Again, for $t$ small enough, these zeros will still be distinct.

3.2. Some matrix inner functions. We now construct a family of $2 \times 2$ matrixvalued analytic functions which are unimodular on the boundary $B$ and have precisely 6 zeros in $R$ starting from certain positive $2 \times 2$ matrix-valued harmonic functions on $R$. This will later provide the basis for our counterexample. 
For our purposes, a matrix-valued $F: R \rightarrow M_{2}(\mathbb{C})$ has simple zeros in $R$ if $Z=\left\{a_{1}, \ldots, a_{n}\right\}$, the zeros of $\operatorname{det}(F)$, have multiplicity either one or two, and if the zero $z \in Z$ has multiplicity two, then $F(z)=0$.

Definition 3.6. We say that $F$ has a standard zero set if $F$ has distinct simple zeros $0, a_{1}, \ldots, a_{4} \in \mathbb{R}$, with the first having multiplicity two and the others multiplicity one. If furthermore, $\delta_{j} \neq 0$ are such that $F\left(a_{j}\right)^{*} \delta_{j}=0, j=1, \ldots, 4$, then no three of the $\delta_{j}$ 's are collinear.

While it is not required immediately, we also ask that $J a_{j} \neq P_{k}, k=1,2$, where $P_{1}, P_{2}$ are poles of the Fay kernel $K^{0}(\cdot, z)$ (defined in Section 4). This last assumption plays an important role in the diagonalization arguments in Section 5.8. In this regard it is also useful to know that the points $P_{1}, P_{2}$ are real and distinct (see Lemma 4.1). Also not required immediately but ultimately useful is the assumption that each $\delta_{j}$ is sufficiently close to some $e_{k}$, a standard basis element of $\mathbb{C}^{2}$ - how close being determined by Theorem 4.3 .

We take $t$ and $p(t)$ as at the end of the last section.

Recall $M(p)$ and $\tau(p)$ from Lemma 2.8 For notational purposes, write $p(t)=$ $\left(1, q_{1}, q_{2}\right)$. Let $\tau$ denote the common value of $\tau\left(1, q_{1}, q_{2}\right), \tau\left(1, q_{1}^{*}, q_{2}\right), \tau\left(1, q_{1}, q_{2}^{*}\right)$, and $\tau\left(1, q_{1}^{*}, q_{2}^{*}\right)$.

For $0 \leq \eta \leq 1$, let $P_{+}^{\eta}$ denote the projection onto the span of $\eta e_{1}+\left(1-\eta^{2}\right)^{\frac{1}{2}} e_{2}$ and $P_{-}^{\eta}$ denote the projection onto $\left(1-\eta^{2}\right)^{\frac{1}{2}} e_{1}-\eta e_{2}$ (so $P_{+}^{\eta}+P_{-}^{\eta}=I$ ). Define $H_{\eta, t}$ by

$$
\begin{aligned}
H_{\eta, t}=\tau_{0} \mathbb{P}(\cdot, 1) I+\tau_{1} \mathbb{P}\left(\cdot, c_{1}-r_{1} \exp \left(i t_{1}\right)\right) P_{+}^{1}+\tau_{1} \mathbb{P}\left(\cdot, c_{1}-r_{1} \exp \left(-i t_{1}\right)\right) P_{-}^{1} \\
+\tau_{2} \mathbb{P}\left(\cdot,-c_{2}-r_{2} \exp \left(i t_{2}\right)\right) P_{+}^{\eta}+\tau_{2} \mathbb{P}\left(\cdot,-c_{2}-r_{2} \exp \left(i t_{2}\right)\right) P_{-}^{\eta} .
\end{aligned}
$$

That is, given $x \in \mathbb{C}^{2}$ a unit vector, $\left\langle H_{\eta, t}(\zeta) x, x\right\rangle$ is the positive harmonic function on $B$ corresponding to the measure

$$
\begin{aligned}
\mu_{x, x}=\tau_{0} \delta_{1} & +\tau_{1}\left(\delta_{q_{1}}\left|x_{1}\right|^{2}+\delta_{q_{1}^{*}}\left|x_{2}\right|^{2}\right) \\
& +\tau_{2}\left(\delta_{q_{2}}\left|\eta x_{1}+\left(1-\eta^{2}\right)^{\frac{1}{2}} x_{2}\right|^{2}+\delta_{q_{2}^{*}}\left|\left(1-\eta^{2}\right)^{\frac{1}{2}} x_{1}-\eta x_{2}\right|^{2}\right) .
\end{aligned}
$$

Then

$$
\begin{aligned}
\int_{B} Q_{j} d \mu_{x, x}= & \tau_{0} Q_{j}(1)+\tau_{1}\left(Q_{j}\left(q_{1}\right)\left|x_{1}\right|^{2}+Q_{j}\left(q_{1}^{*}\right)\left|x_{2}\right|^{2}\right) \\
& +\tau_{2}\left(Q_{j}\left(q_{2}\right)\left|\eta x_{1}+\left(1-\eta^{2}\right)^{\frac{1}{2}} x_{2}\right|^{2}+Q_{j}\left(q_{2}^{*}\right)\left|\left(1-\eta^{2}\right)^{\frac{1}{2}} x_{1}-\eta x_{2}\right|^{2}\right) .
\end{aligned}
$$

Using $Q_{j}\left(q_{k}^{*}\right)=Q_{j}\left(q_{k}\right)$ and this choice of $\tau$ gives

$$
\begin{aligned}
\int_{B} Q_{j} d \mu_{x, x}= & \tau_{0} Q_{j}(1)+\tau_{1} Q_{j}\left(q_{1}\right)\left(\left|x_{1}\right|^{2}+\left|x_{2}\right|^{2}\right) \\
& \quad+\tau_{2} Q_{j}\left(q_{2}\right)\left(\left|\eta x_{1}+\left(1-\eta^{2}\right)^{\frac{1}{2}} x_{2}\right|^{2}+\left|\left(1-\eta^{2}\right)^{\frac{1}{2}} x_{1}-\eta x_{2}\right|^{2}\right) \\
= & \tau_{0} Q_{j}(1)+\tau_{1} Q_{j}\left(q_{1}\right)+\tau_{2} Q_{j}\left(q_{2}\right) \\
= & 0 .
\end{aligned}
$$

Hence $\left\langle H_{\eta, t}(\zeta) x, x\right\rangle$ is the real part of an analytic function. It follows that $H_{\eta, t}$ is the real part of an analytic $2 \times 2$ matrix-valued function $G_{\eta, t}(\zeta)$ normalized by $G_{\eta, t}(0)=I$. 
Our desired functions are

$$
\Psi_{\eta, t}=\left(G_{\eta, t}-I\right)\left(G_{\eta, t}+I\right)^{-1} .
$$

Lemma 3.7. For each $\eta$,

(1) $\Psi_{\eta, t}$ is analytic in a neighborhood of $X$ and unitary-valued on $B$;

(2) $\Psi_{\eta, t}(0)=0$;

(3) $\Psi_{\eta, t}(1)=I$;

(4) $\Psi_{\eta, t}\left(q_{1}\right) e_{1}=e_{1}$ and $\Psi_{\eta, t}\left(q_{1}^{*}\right) e_{2}=e_{2}$;

(5) $\Psi_{\eta, t}\left(q_{2}\right)\left(\eta e_{1}+\left(1-\eta^{2}\right)^{\frac{1}{2}} e_{2}\right)=\left(\eta e_{1}+\left(1-\eta^{2}\right)^{\frac{1}{2}} e_{2}\right)$ and $\Psi_{\eta, t}\left(q_{2}^{*}\right)\left(\left(1-\eta^{2}\right)^{\frac{1}{2}} e_{1}-\eta e_{2}\right)=\left(1-\eta^{2}\right)^{\frac{1}{2}} e_{1}-\eta e_{2} ;$

(6) $\Psi_{0, t}=\left(\begin{array}{cc}\psi_{t} & 0 \\ 0 & \psi_{-t}\end{array}\right)$.

Proof. In the neighborhood of a point $p \in B$, the Poisson kernel $\mathbb{P}(\zeta, p)$ is the real part of a function of the form $g_{p}(\zeta)(\zeta-p)^{-1}$ where $g_{p}$ is analytic in a neighborhood of $p$ and does not vanish at $p$. Near any other point $q \in B, \mathbb{P}(\zeta, p)$ extends to a harmonic function in a neighborhood of $q$ (see Fisher [21, chapter 4, proposition $6.4)$. Thus $\mathbb{P}(\zeta, p)$ is, near $q$, the real part of an analytic function, the real part of which is 0 at $q$.

From the above discussion, if $p \in B$ is different from $1, q_{1}, q_{1}^{*}, q_{2}, q_{2}^{*}$, then $G_{\eta, t}$ is, at least locally, analytic in a neighborhood of $p$. Furthermore, $G_{\eta, t}+I$ is invertible near $p$, since $G_{\eta, t}(\zeta)=H_{\eta, t}(\zeta)+i A(\zeta)$, for some self-adjoint matrix-valued function $A(\zeta)$ and $H_{\eta, t}(p)=0$ for such a $p$. Thus, $G_{\eta, t}+I$ is invertible at $p$ and by continuity, also invertible near $p$. The relation

$$
I-\Psi_{\eta, t}(\zeta) \Psi_{\eta, t}(\zeta)^{*}=2\left(G_{\eta, t}(\zeta)+I\right)^{-1}\left(G_{\eta, t}(\zeta)+G_{\eta, t}(\zeta)^{*}\right)\left(G_{\eta, t}(\zeta)+I\right)^{*-1}
$$

now shows that $\Psi_{\eta, t}$ is unitary at $p$.

From the definition of $G_{\eta, t}$, in a neighborhood of 1 , there exist analytic functions $g_{1}, g_{2}, h_{1}$, and $h_{2}$ so that the real parts of $h_{j}$ are 0 at 1 , each $g_{j}$ is different from 0 at 1 , and

$$
G_{\eta, t}(\zeta)=\left(\begin{array}{cc}
\frac{g_{1}}{\zeta-1} & h_{1} \\
h_{2} & \frac{g_{2}}{\zeta-1}
\end{array}\right)
$$

Thus,

$$
\begin{aligned}
\left(G_{\eta, t}(\zeta)+I\right)^{-1} & \\
= & \frac{1}{\frac{g_{1}+\zeta-1}{\zeta-1} \frac{g_{2}+\zeta-1}{\zeta-1}-h_{1} h_{2}}\left(\begin{array}{cc}
\frac{g_{2}+\zeta-1}{\zeta-1} & -h_{1} \\
-h_{2} & \frac{g_{1}+\zeta-1}{\zeta-1}
\end{array}\right) \\
& =\frac{\left(\begin{array}{cc}
\left(g_{2}+\zeta-1\right)(\zeta-1) & -h_{1}(\zeta-1)^{2} \\
-h_{2}(\zeta-1)^{2} & \left(g_{1}+\zeta-1\right)(\zeta-1)
\end{array}\right)}{\left(g_{1}+\zeta-1\right)\left(g_{2}+\zeta-1\right)-h_{1} h_{2}(\zeta-1)^{2}} .
\end{aligned}
$$

Note that the determinant in the denominator is indeed different from 0 near 1 , and as $\zeta \rightarrow 1$, it goes to $g_{1}(1) g_{2}(1) \neq 0$. Hence $G_{\eta, t}+I$ is in fact invertible. Next, by directly computing $\left(G_{\eta, t}-I\right)\left(G_{\eta, t}+I\right)^{-1}=\Psi_{\eta, t}$ we see that $\Psi_{\eta, t}$ is analytic in a neighborhood of 1 and $\Psi_{\eta, t}(1)=I$.

Now move on to $q_{1}$. Near this point there exist analytic functions $g, h_{2}, h_{3}$, and $h_{4}$ so that the real parts of the $h_{j}$ are 0 at $q_{1}$, the function $g$ does not vanish at $q_{1}$ 
and

$$
G_{\eta, t}=\left(\begin{array}{cc}
\frac{g}{\zeta-q_{1}} & h_{2} \\
h_{3} & h_{4}
\end{array}\right)
$$

Since $h_{4}+1$ has real part 1 at $q_{1}$, whereas $g\left(\zeta-q_{1}\right)^{-1}$ has a pole and $h_{2}, h_{3}$ are analytic at $q_{1}$, we see that $G_{\eta, t}+I$ is invertible near $q_{1}$. Furthermore, by direct computation of $\Psi_{\eta, t}=\left(G_{\eta, t}-I\right)\left(G_{\eta, t}+I\right)^{-1}$ we see that $\Psi_{\eta, t}$ is analytic in a neighborhood of $q_{1}$ and

$$
\Psi_{\eta, t}\left(q_{1}\right)=\left(\begin{array}{cc}
1 & 0 \\
0 & \frac{h_{4}\left(q_{1}\right)-1}{h_{4}\left(q_{1}\right)+1}
\end{array}\right) .
$$

The analogous result holds for the point $q_{1}^{*}$. For the points $q_{2}, q_{2}^{*}$, the same argument prevails by writing all matrices with respect to the orthonormal basis $\left\{\eta e_{1}+\left(1-\eta^{2}\right)^{\frac{1}{2}} e_{2}, \eta e_{2}+\left(1-\eta^{2}\right)^{\frac{1}{2}} e_{1}\right\}$ of $\mathbb{C}^{2}$.

Finally, (6) is easily seen from the definition of $H_{0, t}$ and the functions $G_{0, t}$, $\Psi_{0, t}$.

We are able to obtain some information on the zeros of $\Psi_{\eta, t}$, at least for certain $\eta$.

Lemma 3.8. There exists an $\epsilon>0$ such that for all $\eta$ nonzero but less than $\epsilon, \Psi_{\eta, t}$ has a standard zero set.

Proof. Recall from our choice of $t$, the zeros of $\psi_{t}$ are distinct and, aside from 0 , are not real. Of course the zeros of $\psi_{-t}$ are just the complex conjugates of those of $\psi_{t}$. Thus

$$
\Psi_{0}=\left(\begin{array}{cc}
\psi_{t} & 0 \\
0 & \psi_{-t}
\end{array}\right)
$$

has a standard zero set.

By choosing a sequence $\eta_{n}$ converging to 0 and considering the uniformly bounded sequence $\Psi_{n}=\Psi_{\eta_{n}, t}$, there is some subsequence, still written as $\Psi_{n}$, which converges uniformly on compact subsets of $R$ to some $\Psi$. Consequently,

$$
G_{n}=\left(I+\Psi_{n}\right)\left(I-\Psi_{n}\right)^{-1}
$$

converges uniformly on compact subsets of $R$ to

$$
G=(I+\Psi)(I-\Psi)^{-1} .
$$

Now $H_{n}$, the real part of $G_{n}$, is harmonic and

$$
H_{n}-H_{0}=\mathbb{P}\left(\cdot, q_{2}\right)\left(\tau_{2}\left(\eta_{n}\right) P_{+}^{\eta_{n}}-\tau_{2}(0) P_{+}^{0}\right)+\mathbb{P}\left(\cdot, q_{2}^{*}\right)\left(\tau_{2}\left(\eta_{n}\right) P_{-}^{\eta_{n}}-\tau_{2}(0) P_{-}^{0}\right) .
$$

Thus, as both $\left(P_{ \pm}^{\eta_{n}}-P_{ \pm}^{0}\right)$ and $\left(\tau_{2}\left(\eta_{n}\right)-\tau_{2}(0)\right)$ converge to 0 with $n$ ( $\tau$ is continuous by Lemma 2.8), the $H_{n}$ converge to $H_{0}$ uniformly on compact sets. Since also $G(0)=I=G_{0}(0)$, it follows that the $G_{n}$ converge to $G_{0}$ and hence the $\Psi_{n}$ converge uniformly to $\Psi_{0}$ on compact sets.

Let $d_{n}(\zeta)=\operatorname{det}\left(\Psi_{n}(\zeta)\right)$. This is analytic and unimodular on the boundary and $d_{n}$ converges uniformly to $d_{0}$ on compact sets. From Rouche's theorem (or else Hurwitz's theorem - see Conway [17]), the number of zeros of $d_{n}$ is constant, equaling 6 . Indeed, these zeros vary continuously with $n$, and $d_{n}$ has a double zero at 0 . If we let $0, w_{1}, w_{2}$ denote the zeros of $\psi_{t}$, then the zeros of $\psi_{-t}$ are $0, w_{1}^{*}, w_{2}^{*}$. Hence the zeros of $\Psi_{0}$ are exactly given by $\Psi_{0}(0)=0, \Psi_{0}\left(w_{j}\right) e_{1}=0$, and 
$\Psi_{0}\left(w_{j}^{*}\right) e_{2}=0, \ell=1,2$, and, listed as $0, w_{1}, w_{2}, w_{1}^{*}, w_{2}^{*}$, they are distinct. Thus, for large $n$, the zeros $0, a_{1}^{n}, a_{2}^{n}, a_{3}^{n}, a_{4}^{n}$ of $\Psi_{n}$ satisfy this assumption too.

Finally, if $\Psi_{n}\left(a_{1}^{n}\right)^{*} \delta_{1}^{n}=0$ and $a_{1}$ is close to $w_{1}$, then

$$
\Psi_{0}\left(w_{1}\right)^{*} \delta_{1}^{n}=\left(\Psi_{0}\left(w_{1}\right)-\Psi_{0}\left(a_{1}^{n}\right)\right) \delta_{1}^{n}+\left(\Psi_{0}\left(a_{1}^{n}\right)-\Psi_{n}\left(a_{1}^{n}\right)\right) \delta_{1}^{n} .
$$

For $n$ large both terms on the right-hand side are small and thus for $n$ large $\delta$ is close to (a scalar multiple of) $e_{1}$. Thus, for $n$ large enough the $\delta_{j}^{n}$ satisfy the assumption that no three are collinear. Finally, since the zeros vary continuously with $\eta$ and for $\eta=0$ the zeros, aside from 0 , are not real, whereas $P_{1}, P_{2}$ are real (and not zero - see Lemma 4.1), for small enough $\eta$, neither $P_{1}$ nor $P_{2}$ will be a zero of $\Psi_{\eta, t}$ and the nonzero zeros of $\Psi_{\eta, t}$ will be distinct.

This completes the proof.

3.3. The period matrix and Abel-Jacobi map. Since the harmonic measures $h_{j}$ vanish on $B_{0}$, for $j=1,2$, they reflect across $B_{0}$ by

$$
\begin{aligned}
h_{j}\left(1 / z^{*}\right) & =-h_{j}(z), \\
h_{j}(J z) & =-h_{j}(z)
\end{aligned}
$$

to a harmonic function on $Y \backslash B_{j}$. Note, when $R^{\prime}$ is viewed as the reflection of $R$ across $B_{0}, h_{j}$ is -1 on the reflection of $B_{j}$.

While a harmonic conjugate $\tilde{h_{j}}$ of $h_{j}$ is only locally defined, the differential

$$
\frac{d\left(h_{j}+\tilde{h_{j}}\right)}{d z} d z
$$

is uniquely determined and globally defined. If $\zeta$ is a point of $Y$, then

$$
\chi(\zeta)=\frac{1}{2}\left(\begin{array}{l}
\int_{-1}^{\zeta} \frac{d\left(h_{1}+\tilde{h_{1}}\right)}{d z} d z \\
\int_{-1}^{\zeta} \frac{d\left(h_{2}+\tilde{h_{2}}\right)}{d z} d z
\end{array}\right)
$$

depends upon the path of integration from -1 to $\zeta$, but only up to the number of windings of the path around the boundary components $B_{1}, B_{2}$ and the number of crossings of $B_{1}, B_{2}$ (in passing from $R$ to $R^{\prime}$ ). The choice of -1 for the base point is fairly canonical as it is a Weierstrass point for $Y$ (see subsection 3.1).

The multiple-valued nature of $\chi: Y \rightarrow \mathbb{C}^{2}$ is concisely described by the period matrix and period lattice for $Y$. Let

$$
\mathbf{P}_{j, \ell}=\frac{1}{i} \int_{B_{\ell}} \frac{d\left(h_{j}+\tilde{h_{j}}\right)}{d z} d z=\int_{B_{\ell}} \frac{\partial h_{j}}{\partial n} d s .
$$

Thus, $\mathbf{P}_{j, \ell}$ is the period of the harmonic conjugate of $h_{j}$ around $B_{\ell}$. The $2 \times 2$ matrix $\mathbf{P}$ has positive definite real part and is called the period matrix for $R$. (Our period matrix differs by a factor of $1 / i$ from what most call the period matrix.)

Let $L$ denote the lattice $\mathbb{Z}^{2}+i \mathbf{P} \mathbb{Z}^{2}$. The Jacobi variety of $Y$ is the quotient $\operatorname{Jac}(Y)=\mathbb{C}^{2} / L$. Let $[z]$ denote the class of $z \in \mathbb{C}^{2}$ in $\operatorname{Jac}(Y)$. The mapping $\chi_{0}: Y \rightarrow \operatorname{Jac}(Y)$,

$$
\chi_{0}(\zeta)=[\chi(\zeta)]
$$

is well defined and known as the Abel-Jacobi map. However, it will often be convenient to work with $\chi$, the multiple-valued version of $\chi_{0}$.

Let $e_{1}, e_{2}$ be the usual basis for $\mathbb{C}^{2}$.

Proposition 3.9 ([19], p. 92). The Abel-Jacobi map has the following properties:

(1) $\chi_{0}$ is a one-to-one conformal map of $Y$ onto its image in Jac $(Y)$; 
(2) $\chi_{0}(J \zeta)=-\chi_{0}(\zeta)^{*}$;

(3) If $\zeta \in B$, then $-\chi(\zeta)^{*}=\chi(\zeta)+n$ for some $n \in \mathbb{Z}^{2}$.

Property (2) depends upon the choice of basepoint $-1 \in B_{0}$. Property (3) follows from the observation that for $\zeta \in B, h_{j}(\zeta)$ is either 0 or 1 .

3.4. The theta function and its zeros. Details for most of what follows in this subsection can be found in Ch. IV of [19] and Ch. 2 of [24].

Let $L$ be the lattice defined in the last subsection. The Riemann theta function associated to $L$ is the entire function on $\mathbb{C}^{2}$ defined by

$$
\theta(z)=\sum_{n \in \mathbb{Z}^{2}} \exp (-\pi\langle\mathbf{P} n, n\rangle+2 \pi i\langle z, n\rangle),
$$

where $\langle\cdot, \cdot\rangle$ is the usual inner product on $\mathbb{C}^{2}$.

Straightforward manipulations show that $\theta\left(z^{*}\right)=\theta(z)^{*}$ and $\theta(-z)=\theta(z)$.

The quasi-periodic behavior of $\theta$ with respect to $L$ is given by

$$
\begin{aligned}
\theta(z+\ell) & =\theta(z), \\
\theta(z+i \mathbf{P} m) & =\exp (\pi\langle\mathbf{P} m, m\rangle-2 \pi i\langle z, m\rangle) \theta(z),
\end{aligned}
$$

where $\ell, m \in \mathbb{Z}^{2}$.

Given $e \in \mathbb{C}^{2}$ write $e=u+i \mathbf{P} v$, and define the theta function with characteristic $[e]$ by

$$
\theta[e](z)=\theta\left[\begin{array}{l}
u \\
v
\end{array}\right](z)=\exp (\pi i(\langle\mathbf{P} v, v\rangle+2\langle u-z, v\rangle) \theta(z-e)) .
$$

The function $\theta[e]: \mathbb{C}^{2} \rightarrow \mathbb{C}$ obeys the period laws

$$
\begin{aligned}
\theta[e](z+\ell) & =\exp (-2 \pi\langle\ell, v\rangle) \theta[e](z), \\
\theta[e](z+i \mathbf{P} m) & =\exp (2 \pi\langle m, v\rangle) \exp (\pi\langle\mathbf{P} m, m\rangle-2 \pi i\langle z, m\rangle) \theta[e](z) .
\end{aligned}
$$

It turns out that almost all meromorphic functions and differentials on $Y$ can be represented in terms of translates $\theta[e](\chi(\zeta)): Y \rightarrow \mathbb{C}^{2}$, which despite being multiple valued has a well-defined zero set, the description of which is due to Riemann.

Theorem 3.10. There exists a constant vector $\Delta$ (depending upon the choice of basepoint) so that for each $e \in \mathbb{C}^{2}$, either $\theta[e](\chi(\zeta))$ is identically zero, or $\theta[e](\chi(\zeta))$ has exactly 2 zeros $P_{1}, P_{2}$, and

$$
\chi\left(P_{1}\right)+\chi\left(P_{2}\right)=e-\Delta \text { modulo } L .
$$

Here $\Delta$ is known as the vector of Riemann constants.

The set

$$
\mathcal{N}=\{e \in \operatorname{Jac}(Y): \theta(\chi(\zeta)-e) \text { is identically zero }\}
$$

is a proper closed subset of $\operatorname{Jac}(Y)$.

3.5. The prime form. The following fact plays an important role in construction of the prime form and thus multiple-valued meromorphic functions on $Y$ with prescribed poles and zeros. Given $e \in \mathbb{C}^{2}$ such that $\theta(e)=0$, define

$$
\mathcal{E}_{e}(\zeta, \xi)=\theta(\chi(\zeta)-\chi(\xi)-e) .
$$

Theorem 3.11 ([24], Ch. 2, Lemma 3.4). If $e \in \mathbb{C}^{2}, \theta(e)=0$, and $\mathcal{E}_{e}$ is not identically zero, then there exists $P \in Y$ so that for each $\xi \in Y, \xi \neq P$, the zeros of $\theta[e+\chi(\xi)](\chi(\zeta))$, which coincide with the zeros of $\mathcal{E}_{e}(\zeta, \xi)$, are precisely $\xi$ and $P$. 
The following can be found in Mumford [25] (Lemma 1, p. 3.208), but see also 20] and [10.

Theorem 3.12. There exists $e_{*}=\frac{1}{2}(u+i \mathbf{P} v) \in \mathbb{C}^{2}$ such that $2 e_{*}=0$ modulo $L$, $\langle u, v\rangle$ is odd (equal to 1 modulo $2 \mathbb{Z}$ ), and $\mathcal{E}_{e_{*}}$ is not identically zero.

An $e_{*}$ as in this theorem is called a nonsingular odd half period and for the remainder we take $e_{*}=\frac{1}{2}\left(u_{*}+i \mathbf{P} v_{*}\right)$ as fixed. Note that $\theta\left(e_{*}\right)=0$, as the fact that $\left\langle u_{*}, v_{*}\right\rangle$ is an odd integer implies $\theta\left(e_{*}\right)=-\theta\left(-e_{*}\right)$. Indeed, $e_{*}+e_{*}^{*}=2 u_{*} \in \mathbb{Z}^{2}$ so that, using the periodicity of $\theta$, for $z \in \mathbb{C}^{2}$,

$$
\theta\left(z+e_{*}^{*}\right)=\theta\left(z+e_{*}^{*}+2 u_{*}\right)=\theta\left(z-e_{*}\right) .
$$

Hence $\theta\left(e_{*}\right)=\theta\left(-e_{*}\right)$, and so $\theta\left(e_{*}\right)=0$.

Lemma 3.13. There exists a $P \in B$ so that for each $\xi \in R$, the multiple- valued function $\mathcal{E}_{e_{*}}(\zeta, \xi): Y \rightarrow \mathbb{C}$ given by $\mathcal{E}_{e_{*}}(\zeta, \xi)=\theta\left(\chi(\zeta)-\chi(\xi)-e_{*}\right)$ is not identically zero and has zeros at precisely $P$ and $\xi$.

Proof. From Theorem 3.11, there is a $P$ so that either $\mathcal{E}_{e_{*}}(\zeta, \xi)$ is identically zero, or has zeros $P$ and $\xi$. Accordingly, consider the multiple-valued function $g: Y \rightarrow \mathbb{C}$ defined by $g(\zeta)=\theta\left(\chi(\zeta)-\chi(-1)-e_{*}\right)$. Since $[\chi(-1)]=0$, we may assume $\chi(-1)=0$. Thus $g(\zeta)=\theta\left(\chi(\zeta)-e_{*}\right)$ and the fact that $e_{*}$ is nonsingular means $g$ is not identically zero. Hence $g$ has zeros $P$ and -1 .

As $e_{*}+e_{*}^{*} \in \mathbb{Z}^{2}$, there exists an $n \in \mathbb{Z}^{2}$ such that $e_{*}^{*}=-e_{*}+n$. Similarly, as $\chi(J P)=-\chi(P)^{*}$ modulo $L$, there exists $a, b \in \mathbb{Z}^{2}$ so that $\chi(J P)=-\chi(P)^{*}+a+$ $i \mathbf{P} b$. Hence,

$$
\begin{aligned}
g(J P)^{*} & =\theta\left(\chi(J P)-e_{*}\right)^{*} \\
& =\theta\left(-\chi(P)^{*}+a+i \mathbf{P} b-e_{*}\right)^{*} \\
& =\left(\kappa \theta\left(-\chi(P)^{*}-e_{*}\right)\right)^{*} \\
& =\kappa^{*} \theta\left(-\chi(P)-e_{*}^{*}\right) \\
& =\kappa^{*} \theta\left(-\chi(P)+e_{*}-n\right) \\
& =\kappa^{*} \theta\left(-\chi(P)+e_{*}\right) \\
& =\kappa^{*} \theta\left(\chi(P)-e_{*}\right) \\
& =\kappa^{*} g(P),
\end{aligned}
$$

where $\kappa=\exp \left[\pi\langle\mathbf{P} a, a\rangle-2 \pi i\left\langle-\chi(P)^{*}-e_{*}, n\right\rangle\right]$ is nonzero. Thus, $g(J P)=0$. It follows that $J P=P$ or $J P=-1$, in which case $P=-1$. Thus, $J P=P$ and so $P$ is in $B$.

Since $P$ is in $B, \mathcal{E}_{e_{*}}(\cdot, \xi)$ is not identically zero and the lemma follows from Theorem 3.11

Let

$$
\theta_{*}(z)=\theta\left[e_{*}\right](z)=\exp \left[\frac{1}{2} \pi i\left(\left\langle\mathbf{P} v_{*}, v_{*}\right\rangle+\left\langle u_{*}-z, v_{*}\right\rangle\right)\right] \theta\left(z-e_{*}\right) .
$$

If $z, w \in Y$ are different from the $P$ of Lemma 3.13 then

$$
\frac{\theta_{*}(\chi(\zeta)-\chi(z))}{\theta_{*}(\chi(\zeta)-\chi(w))}=e^{\pi i[\chi(z)-\chi(w)]} \frac{\theta\left(\chi(\zeta)-\chi(z)-e_{*}\right)}{\theta\left(\chi(\zeta)-\chi(w)-e_{*}\right)}
$$

is multiple valued, but its zero/pole structure is well defined: it has a zero at $z$ and a pole at $w$. As we shall see, this will play an important role in defining reproducing kernels on $R$ with respect to harmonic measure. 


\section{The FAY KeRnel FUnCtions of $R$}

We now introduce the reproducing kernel $K^{a}$ as found in Fay 20]. The description of these kernels involves the critical points for the Green's function $g(\cdot, a)$ for $R$ at the point $a \in R$. A point $w$ is a critical point if the gradient of $g(\zeta, a)$ is 0 at $\zeta=w$. It is well known that in a region of connectivity $n+1$ there are $n$ of these critical points $([26$, p. 133$)$. Thus, in $R$ there are two. In the sequel we will have use of the following fact about the location of these critical points for the choice $a=0$.

Lemma 4.1. The critical points of the Green's function $g(\zeta, 0)$ are on the real axis, one in each of the intervals, $\left(-1, c_{1}-r_{1}\right)$ and $\left(c_{2}+r_{2}, 1\right)$.

Proof. For notational ease, let $g(\zeta)=g(\zeta, 0)$. From the symmetry of the domain, $\frac{\partial g}{\partial y}=0$ on the $x$-axis in $R$. Since $g$ is 0 at the points -1 and $c_{1}-r_{1}$, Rolle's Theorem implies there is a point $-1<w_{1}<c_{1}-r_{1}$ so that $\frac{\partial g}{\partial x}\left(w_{1}\right)=0$. Thus, the gradient of $g$ is zero at $w_{1}$. Similarly, there is a point $c_{2}+r_{2}<w_{2}<1$ such that the gradient of $g$ at $w_{2}$ is also zero.

For the remainder of the paper, we let $P_{1}=J w_{1}$ and $P_{2}=J w_{2}$, where $w_{1}, w_{2}$ are the critical points for the Green's function for $R$ at $0, g(\cdot, 0)$. Thus, $P_{1}, P_{2} \in R^{\prime}$. These are the points $P_{1}, P_{2}$ which appear in the definition of a standard zero set, Definition 3.6 .

Theorem 4.2. There is a reproducing kernel $K^{a}$ for the Hardy space $\mathbb{H}^{2}\left(R, \omega_{a}\right)$ of functions analytic in $R$ with boundary values in $L^{2}\left(\omega_{a}\right)$, where $\omega_{a}$ is harmonic measure for the point $a$. If $z=a$, then $K^{a}(\zeta, z)=1$. Otherwise, $K^{a}(\zeta, z)$ has precisely the poles $P_{1}(a), P_{2}(a), J z$ (where $J P_{1}(a)$ and $J P_{2}(a)$ are the critical points for the Green's function for $R$ at a), and three zeros in $Y$, one of which is at Ja.

Incidentally, the Hardy space in the theorem corresponds to just one of the two torus parameter families of rank one bundle shifts over $R$ [1]. Indeed, Ball and Clancey [10] give a theta function representation for all of the corresponding reproducing kernels, avoiding the use of the Klein prime form which Fay uses in his formula for the kernel.

The reader who has skipped sections 3.3 3.5 may wish to skip the proof and proceed directly to subsection 4.1 .

Proof of Theorem 4.2. There is an $e \in \operatorname{Jac}(Y)$ so that

$$
\begin{aligned}
& K^{a}(\zeta, z) \\
& =\frac{\theta\left(\chi(\zeta)+\chi(z)^{*}+e\right) \theta\left(\chi(a)+\chi(a)^{*}+e\right) \theta_{*}\left(\chi(a)+\chi(z)^{*}\right) \theta_{*}\left(\chi(\zeta)+\chi(a)^{*}\right)}{\theta\left(\chi(a)+\chi(z)^{*}+e\right) \theta\left(\chi(\zeta)+\chi(a)^{*}+e\right) \theta_{*}\left(\chi(\zeta)+\chi(z)^{*}\right) \theta_{*}\left(\chi(a)+\chi(a)^{*}\right)} .
\end{aligned}
$$

(See Fay [20], Proposition 6.15 and Ball and Clancey, [10].) Straightforward computation using the periodic nature of $\theta$ checks that the right-hand side is invariant under $\chi(\zeta) \mapsto \chi(\zeta)+n+i \mathbf{P} m$ for $m, n \in \mathbb{C}^{2}$ so that $K^{a}(\zeta, z)$ is in fact single valued and meromorphic in $Y$. Furthermore, according to Fay, for $z \in X, K^{a}(\zeta, z)$ is analytic as a function of $\zeta \in R$. 
One readily verifies from (4.1) that $K^{a}(\zeta, a)=1$, so assume $z \neq a$. Now examine the zero/pole structure of the portion of the right side of (4.1) depending on $\zeta$ :

$$
\frac{\theta\left(\chi(\zeta)+\chi(z)^{*}+e\right) \theta_{*}\left(\chi(\zeta)+\chi(a)^{*}\right)}{\theta\left(\chi(\zeta)+\chi(a)^{*}+e\right) \theta_{*}\left(\chi(\zeta)+\chi(z)^{*}\right)} .
$$

From the comments following (3.4), $\theta_{*}\left(\chi(\zeta)+\chi(a)^{*}\right) / \theta_{*}\left(\chi(\zeta)+\chi(z)^{*}\right)$ has a zero at $J a$ and a pole at $J z$. Each of the remaining theta functions has two zeros, which we label $Z_{1}(z), Z_{2}(z)$ for the top term and $P_{1}(a), P_{2}(a)$ for the bottom term. Hence (4.2) (and so (4.1)) has zeros at $Z_{1}(z), Z_{2}(z), J a$ and poles at $P_{1}(a), P_{2}(a), J z$, the latter of which are all in $R^{\prime} \cup B$, since $K^{a}$ is analytic.

If any of the poles and zeros were to cancel, either $K^{a}(\zeta, z)$ is constant or has two poles and two zeros. Since these kernels are linearly independent and $K^{a}(\zeta, a)=1$, the first possibility cannot occur. In the second case we would be left with two poles in $R^{\prime} \cup B$, which by Proposition 3.1 is also impossible unless $J z$ cancels with $J a$ and the other two poles are in $B$. But then $z=a$ and $K^{a}(\zeta, a)=1$, which we have already ruled out. Hence $K^{a}(\zeta, a)$ has order three, with zeros and poles as claimed.

4.1. Application of the theta function representation of $K^{a}$. We assume throughout that for $j=1, \ldots, 4, a_{j} \in B$ are distinct and $0 \neq \delta_{j} \in \mathbb{C}^{2}$ have the property that no three are collinear (the properties of a standard zero set). As usual $e_{1}, e_{2}$ are the standard basis for $\mathbb{C}^{2}$. We write $P_{1}, P_{2}$ for $P_{1}(0), P_{2}(0)$, the poles of $K^{0}(\zeta, z)$.

Let $\mathcal{M}_{\delta}^{0}$ denote the span of

$$
\begin{aligned}
& \left\{K^{0}(\zeta, 0) e_{1}, K^{0}(\zeta, 0) e_{2}, K^{0}\left(\zeta, a_{1}\right) \delta_{1}, \ldots, K\left(\zeta, a_{4}\right) \delta_{4}\right\} \\
= & \left\{e_{1}, e_{2}, K^{0}\left(\zeta, a_{1}\right) \delta_{1}, \ldots, K\left(\zeta, a_{4}\right) \delta_{4}\right\} .
\end{aligned}
$$

Recall that given $p \in \Pi$ there exists an $s \in B_{1} \times B_{2}=\mathbb{T}^{2}$ so that, identifying $s$ with $(1, s), \phi_{s}=\phi_{p}(1)^{*} \phi_{p}$. In particular, the zeros of $\phi_{p}, z_{0}^{s}=0, z_{1}^{s}, z_{2}^{s}$, depend only upon $s$.

Theorem 4.3. Let $a_{1}^{0}, \ldots, a_{4}^{0}$ be points in $R$ so that $P_{1}, P_{2}, J 0, J a_{1}^{0}, J a_{2}^{0}, J a_{3}^{0}, J a_{4}^{0}$ are all distinct. Let $\left\{e_{1}, e_{2}\right\}$ denote the standard basis for $\mathbb{C}^{2}$ and let $\delta_{1}^{0}=\delta_{2}^{0}=e_{1}$ and $\delta_{3}^{0}=\delta_{4}^{0}=e_{2}$. There exists an $\epsilon>0$ so that if $\left|a_{j}^{0}-a_{j}\right|,\left\|\delta_{j}^{0}-\delta_{j}\right\|<\epsilon$, and if

$$
h(\zeta)=\sum c_{j} K^{0}\left(\zeta, a_{j}\right) \delta_{j}+v
$$

does not have poles at $P_{1}, P_{2}$, then $h$ is constant; i.e., each $c_{j}=0$.

Furthermore, if $h \neq 0$ has a representation as in equation (4.3), and if there exist $z_{1}, z_{2} \in B$ (not assumed to be distinct but not both zero) such that

$$
h(\zeta) K^{0}\left(\zeta, z_{k}\right)=\sum c_{j}^{k} K^{0}\left(\zeta, a_{j}\right) \delta_{j}+v_{k},
$$

then $h$ is constant, $z_{1}=a_{j_{1}}$, and $z_{2}=a_{j_{2}}$, the corresponding $\delta_{j_{k}}$ 's may be taken to be equal to $h$, and all the other terms are zero.

Note that the theorem is really a statement about the meromorphic functions $K^{0}\left(\zeta, a_{j}\right)$ on the double $Y$ and so we view $\zeta$ as a local coordinate on $Y$. Indeed, by restricting $\zeta$ to be near either $P_{1}$ or $P_{2}$ it may be assumed that all the points $\zeta, P_{1}, P_{2}, J a_{1}, \ldots, J a_{4}$ are in a single chart $U \subset R^{\prime}(U$ is an open simply connected subset of $Y)$. 
With fixed $a \in R$ distinct from $0, J P_{1}, J P_{2}$, where $P_{1}, P_{2}$, are the poles of the kernel $K^{0}(\zeta, a)$, the residue of the pole of $K^{0}(\zeta, a)$ at $P_{j}$ is given by the value of the analytic function of $\zeta$,

$$
\left(\zeta-P_{j}\right) K^{0}(\zeta, a)
$$

at the point $\zeta=P_{j}$. Let $R_{j}(a)$ denote this residue.

Lemma 4.4. The residue $R_{j}(a)$ varies continuously with a.

Proof. Consider the theta function representation for $K^{0}(\zeta, a)$ from Theorem 4.2 , The function

$$
f(\zeta)=\theta\left(\chi(\zeta)+\chi(0)^{*}+e\right)
$$

is analytic and single valued in $U$. Furthermore, $f(\zeta)$ vanishes to order one at $P_{j}$ and thus can be written as

$$
f(\zeta)=\left(\zeta-P_{j}\right) f_{j}(\zeta)
$$

where $f_{j}$ is analytic in $U$ and $f_{j}\left(P_{j}\right) \neq 0$. Given a set $W \subset U$, let $W^{*}=\left\{z^{*}: z \in\right.$ $W\}$. Choose neighborhoods $V_{j}$ and $W$ of $U$ so that $F: V_{j} \times W^{*} \rightarrow \mathbb{C}$ by

$$
\begin{aligned}
& F\left(\zeta, a^{*}\right)=f(\zeta) K^{0}(\zeta, a) \\
& \quad=\frac{\theta\left(\chi(\zeta)+\chi(z)^{*}+e\right) \theta\left(\chi(0)+\chi(0)^{*}+e\right) \theta_{*}\left(\chi(0)+\chi(z)^{*}\right) \theta_{*}\left(\chi(\zeta)+\chi(0)^{*}\right)}{\theta\left(\chi(0)+\chi(z)^{*}+e\right) \theta_{*}\left(\chi(\zeta)+\chi(z)^{*}\right) \theta_{*}\left(\chi(0)+\chi(0)^{*}\right)}
\end{aligned}
$$

is analytic. Rewriting gives

$$
\left(\zeta-P_{j}\right) K^{0}(\zeta, a)=\frac{F(\zeta, a)}{f_{j}(\zeta)}
$$

The lemma now follows from the fact that the right-hand side is analytic in $\left(\zeta, a^{*}\right) \in$ $V_{j} \times W$.

Proof of Theorem 4.3. Without loss of generality, we can assume $\epsilon>0$ is small enough that the points $P_{1}, P_{2}, J a_{1}, \ldots, J a_{4}$ are all distinct. Define

and

$$
R\left(a_{1}, a_{2}\right)=\left(\begin{array}{ll}
R_{1}\left(a_{1}\right) & R_{1}\left(a_{2}\right) \\
R_{2}\left(a_{1}\right) & R_{2}\left(a_{2}\right)
\end{array}\right)
$$

$$
R\left(a_{3}, a_{4}\right)=\left(\begin{array}{ll}
R_{1}\left(a_{3}\right) & R_{1}\left(a_{4}\right) \\
R_{2}\left(a_{3}\right) & R_{2}\left(a_{4}\right)
\end{array}\right)
$$

where $R_{j}(a)$ is the residue of $K^{0}(\zeta, a)$ at $P_{j}$ as in Lemma 4.4

To prove that $R\left(a_{1}, a_{2}\right)$ is invertible, let

$$
c=\left(\begin{array}{l}
c_{1} \\
c_{2}
\end{array}\right)
$$

and $f_{c}=c_{1} K^{0}\left(\zeta, a_{1}\right)+c_{2} K^{0}\left(\zeta, a_{2}\right)$. Note that $R\left(a_{1}, a_{2}\right) c=0$ if and only if $f_{c}$ does not have poles at either $P_{1}$ or $P_{2}$. In this case, if $f_{c}$ is not constant, then the poles of $f_{c}$ are precisely the points $J a_{1}$ and $J a_{2}$, which gives the usual contradiction, since both of these points are in $R^{\prime}$. Thus, $f_{c}$ is constant. The kernel $K^{0}(\zeta, 0)=1$, so we can express this as

$$
0=c_{0} K^{0}(\zeta, 0)+c_{1} K^{0}\left(\zeta, a_{1}\right)+c_{2} K^{0}\left(\zeta, a_{2}\right) .
$$

Since $0, a_{1}, a_{2}$ are distinct, the functions $K^{0}(\zeta, 0), K^{0}\left(\zeta, a_{1}\right), K^{0}\left(\zeta, a_{2}\right)$ are linearly independent and hence $c_{1}=c_{2}=0$. Summarizing, if $R\left(a_{1}, a_{2}\right) c=0$, then $c=0$. 
It follows that $R\left(a_{1}, a_{2}\right)$ is invertible and by an identical argument, $R\left(a_{3}, a_{4}\right)$ is invertible.

Consider the function $F$ defined for $\delta_{j}$ near $\delta_{j}^{0}$ by

$$
F=\left(\begin{array}{llll}
R_{1}\left(a_{1}\right) \delta_{1} & R_{1}\left(a_{2}\right) \delta_{2} & R_{1}\left(a_{3}\right) \delta_{3} & R_{1}\left(a_{4}\right) \delta_{4} \\
R_{2}\left(a_{1}\right) \delta_{1} & R_{2}\left(a_{2}\right) \delta_{2} & R_{2}\left(a_{3}\right) \delta_{3} & R_{2}\left(a_{4}\right) \delta_{4}
\end{array}\right) .
$$

Thus, $F$ takes values in $M_{4}$, the $4 \times 4$ matrices, viewed as $2 \times 4$ matrices with entries from $\mathbb{C}^{2}$. Clearly $F$ is continuous in $\delta_{j}$. By Lemma 4.4 it is also continuous in $a_{1}, \ldots, a_{4}$. Indeed, from the form of $F$ it is jointly continuous in $a_{j}, \delta_{j}$. Since $F$ is invertible at $a_{j}^{0}, \delta_{j}^{0}$, it follows that there is an $\epsilon>0$ so that if $\left|a_{j}^{0}-a_{j}\right|,\left\|\delta_{j}^{0}-\delta_{j}\right\|<\epsilon$, then $F$ is invertible.

If $a_{j}$ and $\delta_{j}$ are chosen such that $F$ is invertible and

$$
h(\zeta)=\sum c_{j} K^{0}\left(\zeta, a_{j}\right) \delta_{j}+v
$$

does not have poles at $P_{j}$, then

$$
\begin{aligned}
0 & =\left(\begin{array}{l}
\sum c_{j} R_{1}\left(a_{j}\right) \delta_{j} \\
\sum c_{j} R_{2}\left(a_{j}\right) \delta_{j}
\end{array}\right) \\
& =F\left(\begin{array}{l}
c_{1} \\
c_{2} \\
c_{3} \\
c_{4}
\end{array}\right) .
\end{aligned}
$$

Hence $c=0$ and $h$ is constant.

Now suppose $h \neq 0$ and there exist $z_{1}, z_{2} \in B$ (not assumed distinct but not both zero) such that

$$
h(\zeta) K^{0}\left(\zeta, z_{k}\right)=\sum c_{j}^{k} K^{0}\left(\zeta, a_{j}\right) \delta_{j}+v_{k}, \quad k=0,1,2,
$$

where $z_{0}=0$ (and so $K^{0}\left(\zeta, z_{0}\right)=1$ ). Using $k=1$ we see that $P_{1}, P_{2}$ are not poles of $h$, since by the assumptions on the distinctness of the $P_{k}$ 's and $a_{j}$ 's, the right side has a pole of order at most one at each $P_{k}$, while the left side has a pole of order at least one at these points. Using $k=0$ we see that $h$ satisfies the hypothesis of the part of the theorem which has already been proved. Thus $h$ is constant.

The rest of the result now easily follows using the linear independence of the kernels.

\section{REPRESENTING NICE MATRIX-VALUED INNER FUNCTIONS}

5.1. Hahn-Banach separation. Recall that $\mathbb{H}(X)$ denotes the set of functions analytic in a neighborhood of $X$ and $\mathcal{R}(X)$ the rational functions with poles off $X$. Of particular interest is the set $B \mathbb{H}(X)$ consisting of those $f \in \mathbb{H}(X)$ with $\|f\|_{R} \leq 1$.

Let $M_{2}(\mathbb{H}(X))$ denote the $2 \times 2$ matrices with entries from $\mathbb{H}(X)$ and similarly define $M_{2}(\mathcal{R}(X))$. For $f \in \mathbb{H}(X), f^{*}$ denotes its pointwise complex conjugate while for $F \in M_{2}(\mathbb{H}(X)), F^{*}$ is the pointwise adjoint.

For $f, g \in \mathbb{H}(X)$ or $M_{2}(\mathbb{H}(X))$ and $h(z, w)=\sum f(z) g(w)^{*}$ (with only finitely many terms) we use the convention $\sum f(z) g(w)^{*}(T)=\sum f(T) g(T)^{*}$ to define $h(T)=h\left(T, T^{*}\right)$.

Let $\mathcal{C}$ be the cone generated by

$$
\left\{H(z)\left(1-\psi(z) \psi(w)^{*}\right) H(w)^{*}: \psi \in B \mathbb{H}(X), H \in M_{2}(\mathbb{H}(X))\right\} .
$$


Obviously we would get the same set if we were to instead assume that $H$ is a $\mathbb{C}^{2}$-valued function.

Lemma 5.1. If $F \in M_{2}(\mathbb{H}(X))$, then there exists $\rho>0$ such that $I-\rho^{2} F(z) F(w)^{*}$ $\in \mathcal{C}$.

Proof. First, suppose

$$
F=\left(\begin{array}{l}
f \\
g
\end{array}\right) .
$$

Choose $0<\tau$ so large that $\frac{f}{\tau}$ and $\frac{g}{\tau}$ are in $B \mathbb{H}(X)$. Then,

$$
\begin{aligned}
2 \tau^{2} I & -F(z) F(w)^{*}=2\left(\begin{array}{l}
1 \\
0
\end{array}\right)\left(\tau^{2}-f(z) f(w)^{*}\right)\left(\begin{array}{ll}
1 & 0
\end{array}\right) \\
& +2\left(\begin{array}{l}
0 \\
1
\end{array}\right)\left(\tau_{g}^{2}-g(z) g(w)^{*}\right)\left(\begin{array}{ll}
0 & 1
\end{array}\right)+\left(\begin{array}{c}
f(z) \\
-g(z)
\end{array}\right)(1-0)\left(f(w)^{*} \quad g(w)^{*}\right) .
\end{aligned}
$$

Thus $\rho=\frac{1}{\sqrt{2} \tau}$ satisfies the conclusion of the lemma.

For general $F$ write $F(z) F(w)^{*}=G(z) G(w)^{*}+H(z) H(w)^{*}$, where $G$ and $H$ are the first and second columns of $F$ respectively. There exist $\rho_{G}$ and $\rho_{H}$ so that both $I-\rho_{G}^{2} G(z) G(w)^{*}$ and $I-\rho_{H}^{2} H(z) H(w)^{*}$ are in $\mathcal{C}$. With $\rho^{2}=\frac{1}{2} \min \left\{\rho_{G}^{2}, \rho_{H}^{2}\right\}$,

$$
\begin{aligned}
I-\rho^{2} F(z) F(w)^{*} & =I-\rho^{2} G(z) G(w)^{*}-\rho_{2} H(z) H(w)^{*} \\
& =\frac{1}{2}\left(I-\rho_{G}^{2} G(z) G(w)^{*}\right)+\frac{1}{2}\left(I-\rho_{H}^{2} H(z) H(w)^{*}\right) \\
& +\left(\frac{1}{2} \rho_{G}^{2}+\frac{1}{2} \rho_{H}^{2}-\rho^{2}\right) .
\end{aligned}
$$

Each term on the right-hand side is evidently in $\mathcal{C}$. This completes the proof.

Henceforth, for $F \in M_{2}(\mathbb{H}(X))$, we set

$$
\rho_{F}=\sup \left\{\rho>0: I-\rho^{2} F(z) F(w)^{*} \in \mathcal{C}\right\} .
$$

The following theorem is an application of the Hahn-Banach theorem. It is central to our construction.

Theorem 5.2. If there exists a function $F: R \rightarrow M_{2}(\mathbb{C})$ which is analytic in a neighborhood of $X$ and unitary-valued on $B$ such that $\rho_{F}<1$, then there exists a Hilbert space $\mathcal{H}$ and an operator $T \in \mathcal{B}(\mathcal{H})$ having $X$ as a spectral set, but not having a normal B-dilation.

Proof. The proof features a familiar Hahn-Banach separation argument and GNS construction.

From the hypotheses, there exists a $\rho<1$ so that $I-\rho^{2} F(z) F(w)^{*} \notin \mathcal{C}$.

Let $\mathcal{P}$ be the vector space of finite sums

$$
\sum h_{j}(z) g_{j}(w)^{*}
$$

where $h_{j}, g_{j}$ are $\mathbb{C}^{2}$-valued functions analytic in a neighborhood of $X$. Note, $h(z) g(w)^{*}$ is pointwise a $2 \times 2$ matrix.

The cone $\mathcal{C}$ is a convex subset of $\mathcal{P}$ not containing $I-\rho^{2} F(z) F(w)^{*}$ and by Lemma 5.1. $I$ is an internal point of $\mathcal{C}$. Hence, there exists a nonconstant linear functional $\lambda: \mathcal{P} \rightarrow \mathbb{C}$ so that $\lambda \geq 0$ on $\mathcal{C}$ and $\lambda\left(I-\rho^{2} F(z) F(w)^{*}\right) \leq 0$. We have $\lambda(I)>0$, as otherwise, from Lemma 5.1. $\lambda\left(H(z) H(w)^{*}\right)=0$ for all $H$ and hence $\lambda=0$ (see, for example, Holmes [23], $\S 11 . \mathrm{E}$ ). 
Let $\mathbb{H}_{2}(X)$ denote the $\mathbb{C}^{2}$-valued functions analytic in a neighborhood of $X$. For $h, g \in \mathbb{H}_{2}(X)$, define

$$
[h, g]=\lambda\left(h(z) g(w)^{*}\right) .
$$

Since the cone $\mathcal{C}$ contains $h(z) h(w)^{*}$, the form $[\cdot, \cdot]$ is positive semidefinite on $\mathbb{H}_{2}(X)$.

Given $f$ analytic in a neighborhood of $X$, consider the mapping $M_{f}: \mathbb{H}_{2}(X) \rightarrow$ $\mathbb{H}_{2}(X)$ defined by multiplication by $f$ so that $M_{f} g=f g$. Let $C_{f}$ be the infimum over all positive numbers such that $\frac{f}{C_{f}}$ takes values in the closed unit disk. Obviously, $C_{f}=\|f\|_{R}$. For any $g \in \mathbb{C}^{2}$,

$$
\left(C_{f}^{2} g(z)\right)\left(1-\frac{f(z)}{C_{f}} \frac{f(w)^{*}}{C_{f}}\right)\left(C_{f} g(w)\right)^{*}=g(z)\left(C_{f}^{2}-f(z) f(w)^{*}\right) g(w)^{*} \in \mathcal{C} .
$$

Thus,

$$
\begin{aligned}
C_{f}^{2}[g, g]-\left[M_{f} g, M_{f} g\right] & =C_{f}^{2} \lambda\left(g(z) g(w)^{*}\right)-\lambda\left(f(z) g(z) g(w)^{*} f(w)\right) \\
& =\lambda\left(g(z)\left(C_{f}^{2}-f(z) f(w)^{*}\right) g(w)^{*}\right) \geq 0,
\end{aligned}
$$

as $\lambda$ is nonnegative on $\mathcal{C}$. It follows that each $M_{f}$ defines a bounded operator, still denoted by $M_{f}$, on the Hilbert space $\mathcal{H}$ obtained from $\mathbb{H}_{2}(X)$ by modding out $[\cdot, \cdot]$-null vectors and completing. Furthermore, $\left\|M_{f}\right\|=C_{f}$. In particular, with $T=M_{\zeta}$, where $\zeta(z)=z$ is the identity function, the set $X$ is a spectral set for $T$. Here we are using $f(T)=M_{f}$.

To see that $T$ does not have a dilation to a normal operator with spectrum in $X$, it suffices to show that $F(T)$ is not a contraction for the $F$ in the statement of the theorem. To this end, write

$$
F=\left(\begin{array}{ll}
F_{11} & F_{12} \\
F_{21} & F_{22}
\end{array}\right)
$$

so that

$$
F^{t}(T)=\left(\begin{array}{ll}
M_{F_{11}} & M_{F_{21}} \\
M_{F_{12}} & M_{F_{22}}
\end{array}\right),
$$

where $F^{t}$ denotes the pointwise transpose of $F$. Let $e_{1}, e_{2}$ denote the (class of) constant functions, $e_{j}(z)=e_{j}$, as elements of $\mathcal{H}$. Compute

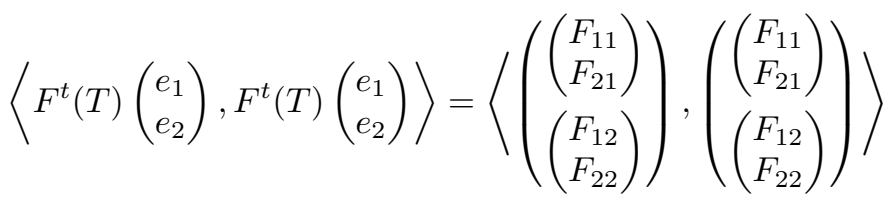

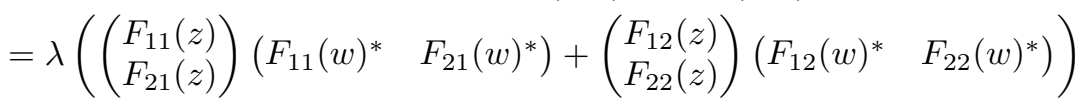

$$
\begin{aligned}
& =\lambda\left(F(z) F(w)^{*}\right) \text {. }
\end{aligned}
$$

On the other hand,

$$
\left\langle\left(\begin{array}{l}
e_{1} \\
e_{2}
\end{array}\right),\left(\begin{array}{l}
e_{1} \\
e_{2}
\end{array}\right)\right\rangle=\lambda\left(e_{1} e_{1}^{*}\right)+\lambda\left(e_{2} e_{2}^{*}\right)=\lambda(I) .
$$

Combining the last two equalities gives

$$
\begin{array}{r}
\left\langle\left(I-F^{t}(T)^{*} F^{t}(T)\right)\left(\begin{array}{l}
e_{1} \\
e_{2}
\end{array}\right),\left(\begin{array}{l}
e_{1} \\
e_{2}
\end{array}\right)\right\rangle=\lambda\left(I-F(z) F(w)^{*}\right) \\
=\lambda\left(\frac{1}{\rho^{2}}-F(z) F(w)^{*}\right)-\left(\frac{1}{\rho^{2}}-1\right) \lambda(I)<0 .
\end{array}
$$


Therefore, $\left\|F^{t}(T)\right\|>1$. On the other hand, $\left\|F^{t}\right\|_{\infty}=\sup \left\{\left\|F^{t}(z)\right\|: z \in X\right\}$ is the same as $\|F\|_{\infty}$, since the norms of a matrix and its transpose are the same. In fact, as $F$ is unitary valued on $B$, so is $F^{t}$ and thus $\left\|F^{t}\right\|_{\infty}=1$. It now follows from Lemma 2.1 that $T$ does not dilate to a normal operator with spectrum in $X$.

5.2. Matrix measures. This subsection is a brief digression from the main line of development to collect some needed facts about matrix-valued measures.

Given a compact Hausdorff space $X$, an $m \times m$ matrix-valued measure

$$
\mu=\left(\mu_{j, \ell}\right)_{j, \ell=1}^{m}
$$

on $X$ is an $m \times m$ matrix whose entries $\mu_{j, \ell}$ are complex-valued regular Borel measures on $X$. The measure $\mu$ is positive, written $\mu \geq 0$, if, for each continuous function $f: X \rightarrow \mathbb{C}^{m}$,

$$
f=\left(\begin{array}{c}
f_{1} \\
\vdots \\
f_{m}
\end{array}\right)
$$

we have

$$
0 \leq \int_{X} f^{*} d \mu f=\sum_{j, \ell} \int_{X} f_{j}^{*} f_{\ell} d \mu_{j, \ell} .
$$

The positive measure $\mu$ is bounded by $C>0$ if

$$
C I_{m}-\left(\mu_{j, \ell}(X)\right) \geq 0
$$

is positive semidefinite, where $I_{m}$ is the identity $m \times m$ matrix.

Lemma 5.3. The $m \times m$ matrix-valued measure $\mu$ is positive if and only if for each Borel set $\omega$ the $m \times m$ matrix

$$
\left(\mu_{j, \ell}(\omega)\right)
$$

is positive semi-definite.

Furthermore, if there is a $\kappa$ so that each diagonal entry $\mu_{j, j}(X) \leq \kappa$, then each entry $\mu_{j, \ell}$ of $\mu$ has total variation at most $\kappa$. In particular, if $\mu$ is bounded by $C$, then each entry has variation at most $C$.

Proof. First suppose $\mu$ is positive. Let $C(X)$ denote the continuous complex-valued functions on $X$. Fix a vector $c \in \mathbb{C}^{m}$. Given $f \in C(X)$, the function $c f$ is a continuous $\mathbb{C}^{m}$-valued function. Thus, $\Phi_{c}: C(X) \rightarrow \mathbb{C}$ given by

$$
\Phi_{c}(f)=\sum \int_{X} c_{j}^{*} c_{\ell} f d \mu_{j, \ell}
$$

is a positive linear functional. Hence $\sum c_{j}^{*} c_{\ell} \mu_{j, \ell}$ is a positive measure on $X$. If $\omega$ is a Borel set, then

$$
\left\langle\left(\mu_{j} \ell(\omega)\right) c, c\right\rangle=\sum c_{j}^{*} c_{\ell} \mu_{j, \ell}(\omega)>0 .
$$

Since $c$ was arbitrary, the matrix in the lemma is positive semidefinite for each $\omega$.

Conversely, suppose the matrix in the lemma is positive semidefinite for each Borel set $\omega$. If $f: X \rightarrow \mathbb{C}^{m}$ is a measurable simple function, $f=\sum v_{j} \chi_{\omega_{j}}$, then

$$
\int_{X} f^{*} d \mu f=\sum_{j}\left\langle\mu\left(\omega_{j}\right) v_{j}, v_{j}\right\rangle \geq 0 .
$$


For more general $f: X \rightarrow \mathbb{C}^{m}$, choose a sequence of measurable simple functions converging to $f$ pointwise. Then,

$$
0 \leq \int_{X} f_{n}^{*} d \mu f_{n} \rightarrow \int_{X} f^{*} d \mu f
$$

Now suppose that each diagonal entry $\mu_{j, j}(X) \leq \kappa$. For $g \in C(X)$, let $\mu_{j, \ell}(g)$ denote the integral of $g$ with respect to $d \mu_{j, \ell}$, and for $j \neq \ell$ let

$$
\lambda=-\frac{\mu_{j, \ell}(g)^{*}}{\left|\mu_{j, \ell}(g)^{*}\right|}\|g\|_{\infty}
$$

Observe

$$
\begin{aligned}
0 & \leq\left\langle\left(\begin{array}{ll}
\mu_{j, j} & \mu_{j, \ell} \\
\mu_{\ell, j} & \mu_{\ell, \ell}
\end{array}\right)\left(\begin{array}{l}
\lambda \\
g
\end{array}\right),\left(\begin{array}{l}
\lambda \\
g
\end{array}\right)\right\rangle \\
& =\|g\|_{\infty}^{2} \mu_{j, j}(X)+\mu_{\ell, \ell}\left(|g|^{2}\right)-2\left|\mu_{j, \ell}(g)\right|\|g\|_{\infty} \\
& \leq 2\left(\|g\|_{\infty}^{2} \kappa-\left|\mu_{j, \ell}(g)\right|\|g\|_{\infty}\right) .
\end{aligned}
$$

It follows that $\mu_{j, \ell}$ is a continuous linear functional on $C(X)$ with norm at most $\kappa$. In particular, the variation of $\mu_{j, \ell}$ is at most $\kappa$.

Finally, by choosing $c=e_{j}$, where $e_{j}$ is the $j$-th standard basis vector for $\mathbb{C}^{m}$, it follows that each $\mu_{j, j}$ is a positive measure. If we now suppose $\mu$ is bounded by $C$, then $\mu_{j, j}(X) \leq C$.

Lemma 5.4. If $\mu^{n}$ is a sequence of positive $m \times m$ matrix-valued measures on $X$ which are all bounded above by $C$, then there is a positive $m \times m$ matrix-valued measure $\mu$ on $X$ also bounded above by $C$. Hence there is a subsequence $\mu^{n_{k}}$ of $\mu^{n}$ converging to $\mu$ weak-*; i.e., for each pair of continuous functions $f, g: X \rightarrow \mathbb{C}^{m}$,

$$
\sum_{j, \ell} \int_{X} f_{\ell} g_{j}^{*} d \mu_{j, \ell}^{n} \rightarrow \sum_{j, \ell} \int_{X} f_{\ell} g_{j}^{*} d \mu_{j, \ell}
$$

Proof. By the previous lemma, for each $j, \ell$ the measures $\mu_{j, \ell}^{n}$ are bounded in variation by $C$. Hence, we can find a subsequence, which for convenience, we will still denote by $\mu^{n}$ so that each $\mu_{j, \ell}^{n}$ converges weak-* to some $\mu_{j, \ell}$ with variation at most $C$.

Suppose $f: X \rightarrow \mathbb{C}^{m}$ is continuous. We have

$$
0 \leq \int_{X} f^{*} d \mu^{n} f=\sum_{j, \ell} \int_{X} f_{j}^{*} f_{\ell} d \mu_{j, \ell}^{n} \rightarrow \sum_{j, \ell} \int_{X} f_{j}^{*} f_{\ell} d \mu_{j, \ell}=\int_{X} f^{*} d \mu f .
$$

Hence $\mu$ is a positive measure. Furthermore, for a vector $c \in \mathbb{C}^{m}$ thought of as a constant function,

$$
0 \leq C\|c\|^{2}-\left\langle\left(\mu_{j, \ell}^{n}(X)\right) c, c\right\rangle \rightarrow C\|c\|^{2}-\left\langle\left(\mu_{j, \ell}(X)\right) c, c\right\rangle .
$$

Thus, $\mu$ is bounded above by $C$.

Lemma 5.5. If $\mu$ is a positive $m \times m$ matrix-valued measure on $X$, then the diagonal entries, $\mu_{j, j}$ are positive measures. Furthermore, with $\nu=\sum_{j=1}^{m} \mu_{j, j}$, there exists an $m \times m$ matrix-valued function $\Delta: X \rightarrow M_{m}(\mathbb{C})$ so that $\Delta(x)$ is positive semidefinite for each $x \in X$ and $d \mu=\Delta d \nu$; i.e., for each pair of continuous functions $f, g: X \rightarrow \mathbb{C}^{m}$,

$$
\sum_{j, \ell} \int_{X} g_{j}^{*} f_{\ell} d \mu_{j, \ell}=\sum_{j, \ell} \int_{X} g_{j}^{*} \Delta_{j, \ell} f_{\ell} d \nu
$$


Proof. From Lemma 5.3 if $\omega$ is a Borel set and $\mu_{j, j}(\omega)=0$, then $\mu_{j, \ell}(\omega)=0$ for each $j$. Thus, for each $j, \ell$ the measure $\mu_{j, \ell}$ is absolutely continuous with respect to $\nu$. By the Radon-Nikodým Theorem, there exists $\nu$ integrable functions $\Delta_{j, \ell}$ so that $d \mu_{j, \ell}=\Delta_{j, \ell} d \nu$.

Once again fix a vector $c \in \mathbb{C}^{m}$. By Lemma 5.3 , for each Borel set $\omega$,

$$
0 \leq\left\langle\left(\mu_{j, \ell}(\omega)\right) c, c\right\rangle=\left\langle\left(\int_{\omega} \Delta_{j, \ell} d \nu\right) c, c\right\rangle=\int_{\omega} \sum_{j, \ell} c_{j}^{*} c_{\ell} \Delta_{j, \ell} d \nu
$$

Thus, $\sum_{j, \ell} c_{j}^{*} c_{\ell} \Delta_{j, \ell} \geq 0$ almost everywhere with respect to $\nu$.

Choose a countable dense subset $\left\{c^{n}\right\}$ of $\mathbb{C}^{m}$. For each $n$ there is a set $E_{n}$ of $\nu$-measure zero such that off of $E_{n}$ the function $\sum_{j, \ell}\left(c_{j}^{n}\right)^{*} c_{\ell}^{n} \Delta_{j, \ell}$ is nonnegative. We have $\sum_{j, \ell}\left(c_{j}^{n}\right)^{*} c_{\ell}^{n} \Delta_{j, \ell}(x) \geq 0$ for each $n$ and for $x \in X \backslash\left(\bigcup E_{n}\right)$. By continuity of the inner product in $\mathbb{C}^{m}$, it follows that $\sum_{j, \ell} c_{j}^{*} c_{\ell} \Delta_{j, \ell}(x) \geq 0$ for all $c \in \mathbb{C}^{m}$ for almost all $x$; that is, the matrices $\Delta(x)=\left(\Delta_{j, \ell}(x)\right)$, for $x \in X \backslash\left(\bigcup E_{n}\right)$, are positive semidefinite.

5.3. Representations in terms of the $\phi_{p}$. The following is a companion to Proposition 5.2. Given a subset $S \subset R$, a function $\Gamma: S \times S \times \Pi \rightarrow \mathbb{C}$ is a positive kernel if for each $p \in \Pi$, the matrix

$$
(\Gamma(z, w ; p))_{z, w \in S^{\prime}}
$$

$S^{\prime}$ a finite subset of $S$. If $S=R$, then $\Gamma$ is analytic if $\Gamma(z, w ; p)$ is analytic in $z$ and conjugate analytic in $w$ for all $p$.

The point of the next proposition is that we do not know a priori that $1-F F^{*} \in$ $\mathcal{C}$ even if $\rho_{F}=1$.

Proposition 5.6. Suppose $F$ is a $2 \times 2$ matrix-valued function analytic in a neighborhood of $R, F$ is unitary-valued on $B$, and $F(0)=0$. If $\rho_{F}=1$ and if $S \subset R$ is a finite set, then there exists a probability measure $\mu$ on $\Pi$ and a positive kernel $\Gamma: S \times S \times \Pi \rightarrow \mathbb{C}$ so that

$$
1-F(z) F(w)^{*}=\int_{\Pi}\left(1-\phi_{p}(z) \phi_{p}(w)^{*}\right) \Gamma(z, w ; p) d \mu(p)
$$

for all $z, w \in S$.

Proof. Choose a sequence $0<\rho_{n}<1$ such that $\rho_{n}$ converges to 1 . For each $n$, there exist vector functions $H_{n, j}$ and functions $\psi_{n, j}$ analytic in a neighborhood of $R$ such that $\psi_{n, j} \in B \mathbb{H}(X)$ and

$$
1-\rho_{n}^{2} F(z) F(w)^{*}=\sum_{j=1}^{N_{n}} H_{n, j}(z)\left(1-\psi_{n, j}(z) \psi_{n, j}(w)^{*}\right) H_{n, j}(w)^{*} .
$$

By post composition with a Möbius transformation if necessary, it may be assumed without loss of generality that $\psi_{n, j}(0)=0$ for all $n, j$.

For each $n, j$, there exist $h_{n, j}(z, p)$, analytic as a function of $z$ in a neighborhood of $R$, and a probability measure $\nu_{n, j}$ on $\Pi$ so that by Proposition 2.4.

$$
1-\psi_{n, j}(z) \psi_{n, j}(w)^{*}=\int_{\Pi} h_{n, j}(z, p)\left(1-\phi_{p}(z) \phi_{p}(w)^{*}\right) h_{n, j}(w, p)^{*} d \nu_{n, j}(p) .
$$

Observe, as all the $\psi$ and $\phi$ vanish at 0 ,

$$
1=\int h_{n, j}(0, p) h_{n, j}(0, p)^{*} d \nu_{n, j}(p) .
$$


Let

$$
\nu_{n}=\sum_{j=1}^{N_{n}} \nu_{n, j} .
$$

By the Radon-Nikodým theorem, there exists a nonnegative function $u_{n, j}(p)$ such that

$$
d \nu_{j, n}=u_{n, j}(p)^{2} d \nu_{n}
$$

Thus

$1-\psi_{n, j}(z) \psi_{n, j}(w)^{*}=\int_{\Pi} u_{n, j}(p) h_{n, j}(z, p)\left[1-\phi_{p}(z) \phi_{p}(w)^{*}\right] h_{n, j}(w, p)^{*} u_{n, j}(p) d \nu_{n}(p)$.

Let

$$
\Gamma_{n}(z, w ; p)=\sum_{j} H_{j, n}(z) u_{n, j}(p) h_{n, j}(z, p) h_{n, j}(w, p)^{*} u_{n, j}(p)^{*} H_{n, j}(w)^{*} .
$$

By construction, $\Gamma_{n}$ is analytic in $z$ and conjugate analytic in $w$ in a neighborhood of $R$, is positive semidefinite as a kernel, and

$$
I-\rho_{n}^{2} F(z) F(w)^{*}=\int_{\Pi}\left[1-\phi_{p}(z) \phi_{p}(w)^{*}\right] \Gamma_{n}(z, w ; p) d \nu_{n}(p) .
$$

For fixed $z, I-\rho_{n}^{2} F(z) F(z)^{*} \leq I$ and there exists an $\epsilon_{z}$ such that $1-\left|\phi_{p}(z)\right|^{2} \geq \epsilon_{z}$. Thus,

$$
I \geq \epsilon_{z} \int_{\Pi} \Gamma_{n}(z, z ; p) d \nu_{n}(p)
$$

Let $C$ denote the maximum of the set $\left\{\epsilon_{z}^{-1}: z \in S\right\}$ and $m$ the cardinality of $S$. The sequence of $m \times m$ measures with $2 \times 2$ entries

$$
d \mu_{n}=\left(\Gamma_{n}(z, w ; p) d \nu_{n}(p)\right)_{z, w \in S}
$$

are positive and the diagonal entries are bounded by $C$. A positive $k \times k$ matrix whose diagonal entries are at most $C$ is bounded above by $k C I_{k}$. It then follows from the results of the previous section that there exists a positive measure $\nu$ and a pointwise positive definite matrix-valued function

$$
\Gamma(p)=(\Gamma(z, w ; p))_{z, w \in S}
$$

so that some subsequence of $\mu_{n}$ converges in the weak-* topology to $\Gamma d \nu$, where $\nu$ can be taken to be a probability measure by scaling $\Gamma$ if necessary. For notational ease, we continue to denote the subsequence by $\mu_{n}$.

For $z, w \in S$ fixed, the expression $\left(1-\phi_{p}(z) \phi_{p}(w)^{*}\right)$ is continuous in $p$ by Lemma 2.11. Thus, letting $n$ tend to infinity in equation (5.2) gives

$$
I-F(z) F(w)^{*}=\int_{\Pi}\left[\left(1-\phi_{p}(z) \phi_{p}(w)^{*}\right] \Gamma(z, w ; p) d \nu(p) .\right.
$$


5.4. Transfer function representations. For present purposes, a unitary colligation $\Sigma=(U, K, \mu)$ consists of a probability measure $\mu$ on $\Pi$, a Hilbert space $K$, and a unitary operator $U$ on the direct sum $\left(L^{2}(\mu) \otimes K\right) \oplus \mathbb{C}^{2}$, written as

$$
U=\left(\begin{array}{ll}
A & B \\
C & D
\end{array}\right)
$$

with respect to the direct sum decomposition. Here $L^{2}(\mu) \otimes K$ signifies $K$-valued $L^{2}(\mu)$.

Define $\Phi: R \rightarrow B\left(L^{2}(\mu) \otimes K\right)$ by $(\Phi(z) f)(p)=\phi_{p}(z) f(p)$. Of course $\Phi$ depends upon $\mu$ and $K$, but this dependence is suppressed. The transfer function associated to $\Sigma$ is

$$
W=W_{\Sigma}(z)=D+C \Phi(z)(I-A \Phi(z))^{-1} B .
$$

Note that as $A$ must be a contraction and $\Phi(z)$ is a strict contraction, the inverse in (5.4) exists for $z \in R$. Moreover, since $\Phi(z)(I-A \Phi(z))^{-1}=(I-\Phi(z) A)^{-1} \Phi(z)$, the transfer function of $\Sigma$ may also be expressed as

$$
W=D+C(I-\Phi(z) A)^{-1} \Phi(z) B .
$$

Proposition 5.7. The transfer function is contraction-valued; that is, $\left\|W_{\Sigma}(z)\right\| \leq$ 1 for all $z \in R$. Indeed, for $z, w \in R$,

$$
I-W_{\Sigma}(z) W_{\Sigma}(w)^{*}=C(I-\Phi(z) A)^{-1}\left(I-\Phi(z) \Phi(w)^{*}\right)(I-\Phi(w) A)^{*-1} C^{*} .
$$

By now the proof is entirely standard. Simply use the equation (5.4), and $D B^{*}=$ $-C A^{*}, B B^{*}=I-A A^{*}$, and $D D^{*}=I-C C^{*}$ to verify:

$$
\begin{aligned}
& I-W(z) W(w)^{*} \\
&= 1-\left[D+C \Phi(z)(I-A \Phi(z))^{-1} B\right]\left[D^{*}+B^{*}\left(I-\Phi^{*}(w) A\right)^{-1} \Phi^{*}(w) C\right] \\
&= 1-D D^{*}-\left[C \Phi(z)(I-A \Phi(z))^{-1} B\right]\left[B^{*}\left(I-\Phi^{*}(w) A^{*}\right)^{-1} \Phi^{*}(w) C\right] \\
&-D B^{*}\left(I-\Phi^{*}(w) A^{*}\right)^{-1} \Phi^{*}(w) C^{*}-C \Phi(z)(I-A \Phi(z))^{-1} B D^{*} \\
&= C\left[1-\Phi(z)(I-A \Phi(z))^{-1}\left(1-A A^{*}\right)\left(I-\Phi^{*}(w) A^{*}\right)^{-1} \Phi^{*}(w)\right. \\
&\left.+A^{*}\left(I-\Phi^{*}(w) A^{*}\right)^{-1} \Phi^{*}(w)+\Phi(z)(I-A \Phi(z))^{-1} A\right] C^{*} \\
&= C\left[1-(I-\Phi(z) A)^{-1} \Phi(z)\left(1-A A^{*}\right) \Phi^{*}(w)\left(I-A^{*} \Phi^{*}(w)\right)^{-1}\right. \\
&\left.+A^{*} \Phi^{*}(w)\left(I-A^{*} \Phi^{*}(w)\right)^{-1}+(I-\Phi(z) A)^{-1} \Phi(z) A\right] C^{*} \\
&= C(I-\Phi(z) A)^{-1}\left[(I-\Phi(z) A)\left(I-A^{*} \Phi^{*}(w)\right)-\Phi(z)\left(1-A A^{*}\right) \Phi^{*}(w)\right. \\
&\left.+(I-\Phi(z) A) A^{*} \Phi^{*}(w)+\Phi(z) A\left(I-A^{*} \Phi^{*}(w)\right)\right]\left(I-A^{*} \Phi^{*}(w)\right)^{-1} C^{*} \\
&= C(I-\Phi(z) A)^{-1}\left[1-\Phi(z) \Phi^{*}(w)\right]\left(I-A^{*} \Phi^{*}(w)\right)^{-1} C^{*} .
\end{aligned}
$$

Note that pointwise on $R$, we can define $H(w)=\left(I-A^{*} \Phi(w)^{*}\right)^{-1} C^{*}: \mathbb{C}^{2} \rightarrow$ $L^{2}(\mu) \otimes K$. Thus, for $w$ fixed, $H(w)^{*}$ is a function on $\Pi$, which we emphasize by writing as $H_{p}(w)^{*}$. Interpreting the representation in Proposition 5.7 in terms of the space $L^{2}(\mu) \otimes K$ gives

$$
I-W(z) W(w)^{*}=\int\left(1-\phi_{p}(z) \phi_{p}(w)^{*}\right) H_{p}(z) H_{p}(w)^{*} d \mu(p) .
$$


5.5. Nevanlinna-Pick interpolation. The following proposition is an Agler-Pick type interpolation theorem for some matrix-valued functions on $R$. The proof proceeds via a transfer function realization for a solution. The now standard methods are purloined from those pioneered by Agler, and followed by Ball and others. We eventually show that, roughly speaking, this theorem only applies to $2 \times 2$ matrix functions which are, up to a fixed unitary, the direct sum of scalar contractive functions.

Proposition 5.8. If $S \subset R$ is a finite set, $W: S \rightarrow M_{2}(\mathbb{C})$, and if there is a positive kernel $\Gamma: S \times S \times \Pi \rightarrow M_{2}(\mathbb{C})$ such that

$$
I-W(z) W(w)^{*}=\int_{\Pi}\left(1-\phi_{p}(z) \phi_{p}(w)^{*}\right) \Gamma(z, w ; p) d \mu(p)
$$

for all $z, w \in S$, then there exists $G: R \rightarrow M_{2}(\mathbb{C})$ such that $\|G(z)\| \leq 1$ and $G(z)=W(z)$ for $z \in S$. Indeed, there exists a finite-dimensional Hilbert space $K$ (dimension at most twice the cardinality of $S$ ) and a unitary colligation $\Sigma=$ $(U, K, \mu)$ so that

$$
G=W_{\Sigma},
$$

and hence there exists $\Delta: R \times R \times \Pi \rightarrow M_{2}(\mathbb{C})$ a positive analytic kernel such that

$$
I-G(z) G(w)^{*}=\int_{\Pi}\left[1-\phi_{p}(z) \phi_{p}(w)^{*}\right] \Delta(z, w ; p) d \mu(p)
$$

for all $z, w \in R$.

Proof. The proof employs a conventional lurking isometry argument. For $p \in \Pi$, the rank of the block matrix with $2 \times 2$ matrix entries

$$
(\Gamma(z, w ; p))_{z, w \in S}
$$

is at most $2 N$, where $N$ is the cardinality of $S$. Thus, by Kolmogorov's theorem (see, for example, 77, Theorem 2.53, especially the second proof), there exists a Hilbert space $K$ of dimension $2 N$ and a function $H: S \rightarrow L^{2}(\mu) \otimes B\left(\mathbb{C}^{2}, K\right)$, denoted $H_{p}(z)$, such that $\Gamma(z, w ; p)=H_{p}(z) H_{p}(w)^{*}$ ( $\mu$ almost everywhere).

Let $\mathcal{E}$ and $\mathcal{F}$ denote the subspaces of $\left(L^{2}(\mu) \otimes K\right) \oplus \mathbb{C}^{2}$ spanned by

$$
\left\{\left(\begin{array}{l}
H_{s}(w)^{*} x \\
W(w)^{*} x
\end{array}\right): x \in \mathbb{C}^{2}, w \in S\right\}
$$

and

$$
\left\{\left(\begin{array}{c}
\phi_{s}(w)^{*} H_{s}(w)^{*} x \\
x
\end{array}\right): x \in \mathbb{C}^{2}, w \in S\right\}
$$

respectively. The mapping $V$ from $\mathcal{E}$ to $\mathcal{F}$ determined by

$$
V\left(\begin{array}{c}
H_{s}(w)^{*} x \\
W(w)^{*} x
\end{array}\right)=\left(\begin{array}{c}
\phi_{s}(w)^{*} H_{s}(w)^{*} x \\
x
\end{array}\right)
$$

is an isometry since

$$
\left\langle\left(\begin{array}{l}
H_{s}(w)^{*} x \\
W(w)^{*} x
\end{array}\right),\left(\begin{array}{l}
H_{s}(z)^{*} y \\
W(z)^{*} y
\end{array}\right)\right\rangle=\left\langle\int H_{s}(z) H_{s}(w)^{*} d \mu(s) x, y\right\rangle+\left\langle W(z) W(w)^{*} x, y\right\rangle
$$


and

$$
\begin{aligned}
& \left\langle\left(\begin{array}{c}
\phi_{s}(w)^{*} H_{s}(w)^{*} x \\
x
\end{array}\right),\left(\begin{array}{c}
\phi_{s}(z)^{*} H_{s}(z)^{*} y \\
y
\end{array}\right)\right\rangle \\
= & \left\langle\int \phi_{s}(z) \phi_{s}(w)^{*} H_{s}(z) H_{s}(w)^{*} d \mu(s) x, y\right\rangle+\langle x, y\rangle .
\end{aligned}
$$

Both $\mathcal{E}$ and $\mathcal{F}$ are finite dimensional, so there exists a unitary $U$ on $\left(L^{2}(\mu) \otimes K\right) \oplus \mathbb{C}^{2}$ such that $U^{*}$ extends $V$. Let $\Sigma=(U, K, \mu)$ denote the resulting unitary colligation.

Write $U$ as in equation (5.3). Since $U^{*}$ restricted to $\mathcal{E}$ is $V$,

$$
\left(\begin{array}{ll}
A^{*} & C^{*} \\
B^{*} & D^{*}
\end{array}\right)\left(\begin{array}{c}
\phi_{s}(w)^{*} H_{s}(w)^{*} x \\
x
\end{array}\right)=\left(\begin{array}{c}
H_{s}(w)^{*} x \\
W(w)^{*} x
\end{array}\right) .
$$

Expressing this as a system of equations we have

$$
\begin{aligned}
& A^{*} \phi_{s}(w)^{*} H_{s}(w)^{*} x+C^{*} x=H_{s}(w)^{*} x, \\
& B^{*} \phi_{s}(w)^{*} H_{s}(w)^{*} x+D^{*} x=W(w)^{*} x .
\end{aligned}
$$

Solving the first equation for $H_{s}(w)^{*} x$ gives

$$
H_{s}(w)^{*} x=\left(I-A^{*} \Phi(w)^{*}\right)^{-1} C^{*} x .
$$

Substituting into this the second equation now gives

$$
B^{*} \Phi(w)^{*}\left(I-A^{*} \Phi(w)^{*}\right)^{-1} C^{*} x=W(w)^{*} x .
$$

It follows that for each $z \in S$,

$$
W_{\Sigma}(z)=W(z)
$$

Next is a uniqueness result for Nevanlinna-Pick interpolation on $R$.

Proposition 5.9. Suppose $F: R \rightarrow M_{2}(\mathbb{C})$ is analytic in a neighborhood of $X$, unitary on $B$, and with a standard zero set. Then there exists $S \subset R$ a set with seven elements such that, if $Z: R \rightarrow M_{2}(\mathbb{C})$ is contraction-valued and $Z(z)=F(z)$ for $z \in S$, then $Z=F$.

Proof. Let $K^{0}$ denote the Fay kernel for $R$ defined in Section 4 . That is, $K^{0}$ is the reproducing kernel for the Hilbert space $\mathbb{H}^{2}(R)$ of functions analytic in $R$ with $L^{2}$ boundary values with respect to harmonic measure on $B$ relative to the point 0 . Let $\mathbb{H}_{2}^{2}(R)$ denote $\mathbb{C}^{2}$-valued $\mathbb{H}^{2}(R)$. Since $F$ is unitary-valued on $B$, the mapping $V$ on $\mathbb{H}_{2}^{2}(R)$ defined by $V G(z)=F(z) G(z)$ is an isometry. As is shown below, the kernel of $V^{*}$ is the span of $\left\{K^{0}\left(\cdot, a_{j}\right) \gamma_{j}: j=1,2, \ldots, 6\right\}$ where $F\left(a_{j}\right)^{*} \gamma_{j}=0$ and, of course, $\gamma_{j} \neq 0$; that is, the pair $\left(a_{j}, \gamma_{j}\right)$ is a zero of $F^{*}$.

Before proceeding, we note that if $\varphi$ is scalar-valued and analytic in a neighborhood of $R$, has no zeros on $B$, and has distinct zeros $w_{1}, \ldots, w_{n} \in R$ of multiplicity one, and if $f \in \mathbb{H}^{2}(R)$ with $f\left(w_{j}\right)=0$, then $f=\varphi g$ for a $g \in \mathbb{H}^{2}(R)$.

Now suppose $\psi \in \mathbb{H}^{2}(R)$ and for all $h \in \mathbb{H}^{2}(R)$, we have $\langle\psi, \varphi h\rangle=0$. Then there is a linear combination $f=\psi-\sum_{1}^{n} c_{j} K^{0}\left(\cdot, w_{j}\right)$ so that $f\left(w_{j}\right)=0$ as the set $\left\{K^{0}\left(\cdot, w_{j}\right): 1 \leq j \leq n\right\}$ is linearly independent, and thus by the above remark, $f=\varphi g$ for some $g \in \mathbb{H}^{2}(R)$. Since $\left\langle K^{0}\left(\cdot, w_{j}\right), \varphi h\right\rangle=\varphi\left(w_{j}\right)^{*} h\left(w_{j}\right)^{*}=0$ for each $j$ and $h$, it follows that $\langle f, \varphi h\rangle=0$ for all $h$. Choosing $h=g$ gives $\langle\varphi g, \varphi g\rangle=0$ from which it follows that $g=0$. Hence $\psi$ is in the span of $\left\{K^{0}\left(\cdot, w_{j}\right): 1 \leq j \leq n\right\}$. 
This shows that $\left\{K^{0}\left(\cdot, w_{j}\right): 1 \leq j \leq n\right\}$ is a basis for the orthogonal complement of $\left\{\varphi h: h \in \mathbb{H}^{2}(R)\right\}$.

We next determine the kernel of $V^{*}$. Write $a_{5}=a_{6}=0$. Since $F(0)=0$, there is a function $H$ analytic in a neighborhood of $X$ so that $F=z H$. The function $\varphi=z \operatorname{det}(H)$ satisfies the hypothesis of the preceding paragraph.

Let

$$
G=\left(\begin{array}{cc}
h_{22} & -h_{12} \\
-h_{21} & h_{11}
\end{array}\right)
$$

where $H=\left(h_{j, \ell}\right)$. Verify $F G=z H G=z \operatorname{det}(H) I$, where $I$ is the $2 \times 2$ identity matrix.

Now suppose $x \in \mathbb{H}_{2}^{2}(R)$ and $V^{*} x=0$. Let $x_{1}, x_{2}$ denote the coordinates of $x$. For each $g \in \mathbb{H}_{2}^{2}(R)$,

$$
0=\left\langle G g, V^{*} x\right\rangle=\langle V G g, x\rangle=\langle z \operatorname{det}(H) g, x\rangle=\left\langle z \operatorname{det}(H) g_{1}, x_{1}\right\rangle+\left\langle z \operatorname{det}(H) g_{2}, x_{2}\right\rangle .
$$

It follows from the discussion above that each $x_{j}$ is in the span of $\left\{K^{0}\left(\zeta, a_{j}\right): 1 \leq\right.$ $j \leq 6\}$ and therefore $x$ is in the span of $\left\{K^{0}\left(\zeta, a_{j}\right) v: 1 \leq j \leq 6, v \in \mathbb{C}^{2}\right\}$. In particular, there exist vectors $v_{j} \in \mathbb{C}^{2}$ such that

$$
x=\sum_{1}^{6} v_{j} K^{0}\left(\cdot, a_{j}\right)
$$

Since, as is readily verified, $V^{*} v K^{0}(\cdot, a)=F(a)^{*} v K^{0}(\cdot, a)$ and $F(0)^{*}=0$,

$$
0=V^{*} x=\sum_{1}^{4} F\left(a_{j}\right)^{*} v_{j} K^{0}\left(\cdot, a_{j}\right) .
$$

But $K^{0}\left(\cdot, a_{j}\right), j=1, \ldots, 4$ are linearly independent, and so $F\left(a_{j}\right)^{*} v_{j}=0$ for each $j$. Conversely, if $F\left(a_{j}\right)^{*} v_{j}=0$, then $V^{*} v_{j} K^{0}\left(\cdot, a_{j}\right)=0$ so that $\left\{K^{0}\left(\zeta, a_{j}\right) v_{j}: 1 \leq\right.$ $j \leq 6\}$ is a basis for the kernel of $V^{*}$.

The projection onto the kernel of $V^{*}$ is $I-V V^{*}$ and since the kernel of $V^{*}$ has dimension six, $I-V V^{*}$ has rank six. Thus, for any finite set $A \subset R$, the block matrix with $2 \times 2$ matrix entries

$$
\begin{aligned}
M_{A} & =\left(\left(\left\langle\left(I-V V^{*}\right) K^{0}(\cdot, w) e_{j}, K^{0}(\cdot, z) e_{\ell}\right\rangle\right)_{j, \ell=1,2}\right)_{z, w \in A} \\
& =\left(\left(I-F(z) F(w)^{*}\right) K^{0}(z, w)\right)_{z, w \in A}
\end{aligned}
$$

has rank at most six. (Here $\left\{e_{1}, e_{2}\right\}$ is the usual basis for $\mathbb{C}^{2}$.) In particular, if $A=\left\{a_{1}, \ldots, a_{6}\right\}$ where the $a_{j}$ are the zeros of $\operatorname{det}(F)\left(\right.$ with $\left.a_{5}=a_{6}=0\right)$, then $M_{A}$ has exactly rank six. Choose points $a_{7}, a_{8}$ distinct from $a_{1}, \ldots, a_{6}$ so that the set $S=\left\{a_{1}, \ldots, a_{6}, a_{7}, a_{8}\right\}$ has exactly seven distinct points. Since $S$ contains $A$, the rank of $M_{S}$ is at least six. On the other hand, by what is proved above, it has rank at most six. Thus the rank of $M_{S}$ is six.

Since the matrix $M_{S}$ is $14 \times 14$ (viewed as a $7 \times 7$ block matrix with $2 \times 2$ matrix entries), and $M_{S}$ has rank six, the kernel of $M_{S}$ has dimension eight. Furthermore, 
as the dimension of the subspace

$$
\left.\mathcal{L}_{1}=\left\{\left(\begin{array}{c}
\left(\begin{array}{c}
\gamma_{1} \\
0
\end{array}\right) \\
\left(\begin{array}{c}
\gamma_{2} \\
0
\end{array}\right) \\
\vdots \\
\left(\gamma_{7}\right. \\
0
\end{array}\right)\right): \gamma=\left(\begin{array}{c}
\gamma_{1} \\
\gamma_{2} \\
\vdots \\
\gamma_{7}
\end{array}\right) \in \mathbb{C}^{7}\right\}
$$

of $\mathbb{C}^{7} \otimes \mathbb{C}^{2}$ has dimension seven, it follows that there exists a nonzero $x_{1}=y_{1} \otimes e_{1}$ in $\mathcal{L}$ and in the kernel of $M_{S}$. Similarly, by considering $\mathcal{L}_{2}=\left\{\gamma \otimes e_{2}: \gamma \in \mathbb{C}^{7}\right\}$, there exists a nonzero $x_{2}$ in the kernel of $M_{S}$ of the form $x_{2}=y_{2} \otimes e_{2}$.

Let $X=\left(\begin{array}{ll}x_{1} & x_{2}\end{array}\right)$; that is, $X$ is a $14 \times 2$ matrix,

$$
X=\left(\begin{array}{c}
\left(\begin{array}{cc}
\left(y_{1}\right)_{1} & 0 \\
0 & \left(y_{2}\right)_{1}
\end{array}\right) \\
\left(\begin{array}{cc}
\left(y_{1}\right)_{2} & 0 \\
0 & \left(y_{2}\right)_{2}
\end{array}\right) \\
\vdots \\
\left(\begin{array}{cc}
\left(y_{1}\right)_{7} & 0 \\
0 & \left(y_{2}\right)_{7}
\end{array}\right)
\end{array}\right) \text {. }
$$

It is convenient to use $S$ to index itself, so that we have

$$
X(w)=\left(\begin{array}{ll}
x_{1}(w) & x_{2}(w)
\end{array}\right)=\left(\begin{array}{cc}
y_{1}(w) & 0 \\
0 & y_{2}(w)
\end{array}\right)
$$

for the $w \in S$ coordinate of $X$. The identity $M_{S} X=0$ becomes

$$
\sum_{w \in S} K^{0}(z, w) X(w)=F(z) \sum_{w \in S} K^{0}(z, w) F(w)^{*} X(w)
$$

for each $z \in S$.

Now suppose $Z: R \rightarrow M_{2}(\mathbb{C})$ is analytic, contraction-valued, and $Z(z)=F(z)$ for $z \in S$. The operator $W$ of multiplication by $Z$ on $\mathbb{H}_{2}^{2}(R)$ is a contraction and

$$
W^{*} K^{0}(\cdot, w) x=Z(w)^{*} x K^{0}(\cdot, w) .
$$

Given $\zeta \in R, \zeta \notin S$, let $S^{\prime}=S \cup\{\zeta\}$ and consider the decomposition of

$$
N_{\zeta}=\left(\left(\left\langle\left(I-Z(z) Z(w)^{*}\right) K^{0}(\cdot, w) e_{j}, K^{0}(\cdot, z) e_{\ell}\right\rangle\right)_{j, \ell}\right)_{z, w \in S^{\prime}}
$$

into blocks labelled by $S$ and $\{\zeta\}$. Thus, $N_{\zeta}$ is an $8 \times 8$ matrix with $2 \times 2$ block entries. The upper left $7 \times 7$ block matrix, the block determined by $S$, is $M_{S}$ since $Z(z)=F(z)$ for $z \in S$. Let

$$
Y=\left(\left(\begin{array}{ll}
0 & 0 \\
0 & 0
\end{array}\right)\right)
$$

Since $N_{\zeta}$ is positive semi-definite and $M_{S} X=0$, it follows that $N_{\zeta} Y=0$. An examination of the last two entries (the last $2 \times 2$ block) of the product $N_{\zeta} Y=0$ 
gives

$$
\sum_{w \in S} K^{0}(\zeta, w) X(w)=Z(\zeta) \sum_{w \in S} Z(w)^{*} K^{0}(\zeta, w) X(w)
$$

From the form of $X$ and since the kernel functions $\left\{K^{0}(\cdot, w): w \in S\right\}$ form a linearly independent set in $\mathbb{H}^{2}\left(R, \omega_{0}\right)$, it follows that the right-hand side in (5.6) has rank two for all but at most countably many $\zeta$. Hence,

$$
\sum_{w \in S} K^{0}(z, w) F(w)^{*} X(w)
$$

also has rank two and $Z$ is completely determined by the identity (5.6). Since $F$ is also determined by this identity, $Z=F$.

The fact that we used $K^{0}$ in the last proof is not significant: any reproducing kernel would have worked. However, certain facts that came to light in the proof will play a role in the proof of Lemma [5.11, where Fay kernels are needed.

5.6. A tight representation. The results in the previous sections now combine to produce a tight representation for $F: R \rightarrow M_{2}(\mathbb{C})$ which is analytic across the boundary, unitary on the boundary, has at most six simple zeros in $R$, and has $\rho_{F}=1$.

Theorem 5.10. Suppose $F$ is a $2 \times 2$ matrix-valued function analytic in a neighborhood of $R$, which is unitary-valued on $B$ and with a standard zero set. If $\rho_{F}=1$, then there exists a unitary colligation $\Sigma=(U, K, \mu)$ such that $F=W_{\Sigma}$ and so that the dimension of $K$ is at most 14 . In particular, $\mu$ is a probability measure on $\Pi$, and there is an analytic function $H: R \rightarrow L^{2}(\mu) \otimes M_{14,2}(\mathbb{C})$, denoted $H_{p}(z)$ so that

$$
1-F(z) F(w)^{*}=\int_{\Pi}\left(1-\phi_{p}(z) \phi_{p}(w)^{*}\right) H_{p}(z) H_{p}(w)^{*} d \mu(p)
$$

for all $z, w \in R$.

Proof. Choose, using Proposition [5.9, a finite set $S$ of $R$ with the property that if $G: R \rightarrow M_{2}(\mathbb{C})$ is analytic, contraction-valued, and $G(z)=F(z)$ for $z \in S$, then $G=F$.

Using this $S$, Proposition 5.6 produces a probability measure $\mu$ and positive kernel $\Gamma: S \times S \times \Pi \rightarrow M_{2}(\mathbb{C})$ such that

$$
1-F(z) F(w)^{*}=\int_{\Pi}\left(1-\phi_{p}(z) \phi_{p}(w)^{*}\right) \Gamma(z, w ; p) d \mu(p)
$$

for all $z, w \in S$.

By Proposition 5.8 there exists a unitary colligation $\Sigma=(U, K, \mu)$ so that $W_{\Sigma}(z)=F(z)$ for $z \in S$. From our choice of $S$, we see that $F=W_{\Sigma}$. The integral representation follows.

5.7. Fay kernels reprise. Suppose $F$ satisfies the hypothesis of Theorem 5.10 and let $\mu$ denote the measure which appears in the conclusion.

Recall Fay's variant of the Szegö kernel for $R, K^{0}(\zeta, z)$. Also recall, for each $p \in \Pi$ there is an $s \in\{1\} \times B_{1} \times B_{2}$ so that $\phi_{s}(z) \phi_{s}(w)^{*}=\phi_{p}(z) \phi_{p}(w)^{*}$. In this case we label the zeros of $\phi_{p}$ (which of course are the same as those of $\phi_{s}$ ) $z_{0}^{s}(=0), z_{1}^{s}, z_{2}^{s}$. 
Lemma 5.11. Suppose $F$ satisfies the hypothesis of Theorem [5.10; $F$ is a $2 \times 2$ matrix-valued function analytic in a neighborhood of $R$ which is unitary-valued on $B$, with a standard zero set, and $\rho_{F}=1$. Assume furthermore that it is represented as in the conclusion of Theorem 5.10. Let $a_{5}=a_{6}=0$ and $\delta_{5}=e_{1}$ and $\delta_{6}=$ $e_{2}$. Then there exists a set $E$ of $\mu$ measure zero such that for $p \notin E$, for each $v \in \mathbb{C}^{14}$ and for $\ell=0,1,2$, the vector function $H_{p}(\zeta) v K^{0}\left(\zeta, z_{\ell}\right)$ is in the span of $\left\{K^{0}\left(\zeta, a_{j}\right) \delta_{j}\right\}$, where $z_{0}(=0), z_{1}, z_{2}$ are the zeros of $\phi_{p}$. As a consequence, $H_{p}$ is analytic on $R$ and extends to a meromorphic function on $Y$.

Proof. Given a finite set $Q \subset R$,

$$
M_{Q}=\left(\left(I-F(z) F(w)^{*}\right) K^{0}(z, w)\right)_{z, w \in Q}
$$

has rank at most six. Moreover, as interpreted in the proof of Proposition 5.9, the range of $M_{Q}$ lies in the span of $\left\{\left(K^{0}\left(z, a_{j}\right) \delta_{j}\right)_{z \in Q}: j=1, \ldots, 6\right\}$. Here $\left(K^{0}\left(z, a_{j}\right) \delta_{j}\right)_{z \in Q}$ is a column vector indexed by $Q$.

From the representation for $F$ of Theorem 5.10 .

$$
M_{Q}=\left(\int H_{p}(z)\left(1-\phi_{p}(z) \phi_{p}(w)^{*}\right) K^{0}(z, w) H_{p}(w)^{*} d \mu(p)\right)_{z, w \in Q} .
$$

For each $p$, multiplication by $\phi_{p}$, denoted $M_{p}$, is isometric on $\mathbb{H}^{2}\left(R, \omega_{0}\right)$, the Hilbert space of analytic functions on $R$. Hence $1-M_{p} M_{p}^{*} \geq 0$, as is $\left(I-M_{p} M_{p}^{*}\right) \otimes E$, where $E$ is the $n \times n$ matrix consisting of all ones. From the reproducing property of $K^{0}(\cdot, z), M_{p}^{*} K^{0}(\cdot, z)=\phi_{p}(z)^{*} K^{0}(\cdot, z)$. Thus, if $Q$ is a set of $n$ points in $R$ and $x$ is the vector $\left(K^{0}(\cdot, w)\right)_{w \in Q}$, then the matrix

$$
P_{Q}(p)=\left\langle\left(\left(I-M_{p} M_{p}^{*}\right) \otimes E\right) x, x\right\rangle=\left(\left(1-\phi_{p}(z) \phi_{p}(w)^{*}\right) K^{0}(z, w)\right)_{z, w \in Q} \geq 0 .
$$

If we set $\tilde{Q}=Q \cup\left\{z_{j}(s)\right\}$, for any $j=0,1$, or 2 , then $P_{\tilde{Q}}(p) \geq 0$ as well. Furthermore, the upper left $n \times n$ block equals $P_{Q}(p)$ and the right $n \times 1$ column is $\left(K_{0}\left(z, z_{j}(s)\right)\right)_{z \in Q}$. Hence as a vector $\left(K_{0}\left(z, z_{j}(s)\right)\right)_{z \in Q} \in \operatorname{ran} P_{Q}(p)^{1 / 2}=\operatorname{ran} P_{Q}(p)$ for $j=0,1,2$.

Since $P_{Q}(p) \geq 0$,

$$
N_{Q}(p)=\left(H_{p}(z)\left(1-\phi_{p}(z) \phi_{p}(w)^{*}\right) K^{0}(z, w) H_{p}(w)^{*}\right)_{z, w \in Q}
$$

is also positive semidefinite for each $p$. If $M_{Q} x=0$, then

$$
0=\int\left\langle N_{Q}(p) x, x\right\rangle d \mu(p),
$$

so that $\left\langle N_{Q}(p) x, x\right\rangle=0$ for almost every $p$. It follows that $N_{Q}(p) x=0$ almost everywhere. Choosing a basis for the kernel of $M_{Q}$, there is a set $E_{Q}$ of measure zero so that for $p \notin E_{Q}$, the kernel of $M_{Q}$ is a subspace of the kernel of $N_{Q}(p)$. Thus, for such $p$, the range of $N_{Q}(p)$ is a subspace of the range of $M_{Q}$. In particular, the rank of $N_{Q}(p)$ is at most 6 .

Moreover, if we let $D_{Q}(p)$ denote the $(2 \times 14$ block $)$ diagonal matrix with $(z, z)$ entry $H_{p}(z)(z \in Q)$, then $N_{Q}(p)=D_{Q}(p) P_{Q}(p) D_{Q}(p)^{*}$. Since $P_{Q}(p)$ is positive semidefinite, we conclude that the range of $D_{Q}(p) P_{Q}(p)$ is a subspace of $M_{Q}$. Thus, as $\left(K^{0}\left(z, z_{j}(s)\right)\right)_{z \in Q}$ is in the range of $P_{Q}(p),\left(H_{p}(z) v K^{0}\left(z, z_{j}(s)\right)\right)_{z \in Q}$ is in the range of $M_{Q}$ for every $v \in \mathbb{C}^{14}$ and $j=0,1,2$. 
Now suppose $Q_{n} \subset R$ is a finite set with $Q_{n} \subset Q_{n+1}, Q_{0}=\left\{a_{1}, \ldots, a_{4}, a_{5}(=0)\right\}$, and $\mathcal{D}=\bigcup_{n} Q_{n}$ a determining set. Then since

$$
\left(H_{p}(z) v K^{0}\left(z, z_{j}(s)\right)\right)_{z \in Q_{n}} \in \operatorname{ran} M_{Q_{n}} \subseteq \bigvee_{j}\left(K^{0}\left(z, a_{j}\right) \delta_{j}\right)_{z \in Q}
$$

there are constants $c_{j}^{n}(p)$ such that

$$
H_{p}(z) v K^{0}\left(z, z_{j}(s)\right)=\sum_{j=1}^{5} c_{j}^{n}(p) K^{0}\left(z, a_{j}\right) \delta_{j}, \quad z \in Q_{n} .
$$

By linear independence of the $K^{0}\left(\cdot, a_{j}\right)$ 's, the $c_{j}^{n}(p)$ 's are uniquely determined when $n=0,1, \ldots$ by (5.8). Since $Q_{n+1} \supset Q_{n}$, we must in this case have $c_{j}^{n+1}(p)=c_{j}^{n}(p)$ for all $n$, and thus there are unique constants $c_{j}(p)$ such that

$$
H_{p}(z) v K^{0}\left(z, z_{j}(s)\right)=\sum_{j=1}^{5} c_{j}(p) K^{0}\left(z, a_{j}\right) \delta_{j}, \quad z \in \mathcal{D}, \quad p=0,1,2 .
$$

By considering this equation with $j=5$, and using the fact that $K^{0}(z, 0)=1$, we see that $H_{p}$ agrees with an analytic function on the determining set $\mathcal{D}$. It follows that we can assume that $H_{p}(z)$ is analytic for each $p \notin E$ and that the relation of equation (5.8) holds throughout $R$. Furthermore, since the $K^{0}\left(\cdot, a_{j}\right)$ 's extend to meromorphic functions on $Y, H_{p}$ does as well.

5.8. Diagonalization. In this subsection we show that when $\rho_{F}=1$, a contractive matrix-valued function with unitary boundary values and a standard zero set is diagonalizable.

Lemma 5.12. Suppose $F$ is a $2 \times 2$ matrix-valued function on $R$ whose determinant is not identically zero. If there exists a $2 \times 2$ unitary matrix $U$ and scalar-valued functions $\psi_{1}, \psi_{2}: R \rightarrow \mathbb{C}$ such that $F(z) F(w)^{*}=U D(z) D(w)^{*} U^{*}$, where

$$
D=\left(\begin{array}{cc}
\psi_{1} & 0 \\
0 & \psi_{2}
\end{array}\right)
$$

then there exists a unitary matrix $V$ so that $F=U D V$.

Proof. The hypotheses imply $D(z)^{-1} U^{*} F(z)=D(w)^{*} U^{*} F(w)^{*-1}$ whenever $F(z)$ and $F(w)$ are invertible. Hence, $D(z)^{-1} U^{*} F(z)=V$ is constant. One readily verifies that $V^{*} V=I$.

Theorem 5.13. Suppose $F$ is a $2 \times 2$ matrix-valued function which is analytic in a neighborhood of $R$, unitary-valued on $B$ and has a standard zero set, $\delta_{j}, a_{j}$, $j=1, \ldots, 4$. Assume further that the $\delta_{j}, a_{j}$ have the property that if $h$ satisfies

$$
h(\zeta)=\sum_{1}^{4} c_{j} K^{0}\left(\zeta, a_{j}\right) \delta_{j}+v
$$

for some $c_{1}, \ldots, c_{4} \in \mathbb{C}$ and $v \in \mathbb{C}^{2}$ and if $h$ does not have pole at either $P_{1}$ or $P_{2}$, then $h$ is constant. 
Under these conditions, if $\rho_{F}=1$, then $F$ is diagonalizable; i.e., there exist $2 \times 2$ unitary matrices $U$ and $V$ and analytic functions $\varphi_{j}: R \rightarrow \mathbb{C}$ such that, with

$$
D=\left(\begin{array}{cc}
\varphi_{1} & 0 \\
0 & \varphi_{2}
\end{array}\right)
$$

$F=V D U$

Proof. By Lemma 5.11 we may assume that, except perhaps on a set $\Delta_{0}$ of measure 0 , if $h$ is a column of some $H_{p}$, then $h(\zeta) K^{0}\left(\zeta, z_{\ell}(s)\right)$ is in the span of $\left\{K^{0}\left(\zeta, a_{j}\right) \delta_{j}\right.$ : $1 \leq j \leq 6\}$ for $\ell=0,1,2$. Here $z_{0}(p)=0, z_{1}(p), z_{2}(p)$ are the zeros of $\phi_{p}$. By hypothesis, $h$ (and so $H_{p}$ ) is constant as a function of $\zeta$. From our normalization of the domain (see Corollary 2.13), one of the zeros of $\phi_{p}$, say $z_{1}(p)$, is not zero. Thus, Theorem 4.3 (or rather the argument there) also implies, that if $h$ is not zero, then there is a $1 \leq j_{1}(p) \leq 4$ so that $z_{1}(p)=a_{j_{1}(p)}$ and $h$ is a multiple of $\delta_{j_{1}(p)}$. Thus, every column of $H_{p}$ is a multiple of $\delta_{j_{1}(p)}$.

Theorem 5.10 gives us the representation

$$
1-F(z) F(w)^{*}=\int_{\Pi}\left(1-\phi_{p}(z) \phi_{p}(w)^{*}\right) H_{p} H_{p}^{*} d \mu(p) .
$$

Substituting $w=0$ we find

$$
I=\int H_{p} H_{p}^{*} d \mu(p)
$$

In view of equation (5.11), rearranging equation (5.10) gives

$$
F(z) F(w)^{*}=\int_{\Pi} \phi_{p}(z) \phi_{p}(w)^{*} H_{p} H_{p}^{*} d \mu(p) .
$$

Since the columns of $H_{p}$ are all multiples of the single vector $\delta_{j_{1}(p)}, H_{p} H_{p}^{*}$ is rank one and thus may be written as $G(p) G(p)^{*}$, for a vector $G(p) \in \mathbb{C}^{2}$. (Indeed, $G(p)$ is the square root of the sum of the squares of the norms of the columns of $H_{p}$ times $\delta_{j_{1}(p)}$.) Consequently,

$$
F(z) F(w)^{*}=\int_{\Pi} \phi_{p}(z) \phi_{p}(w)^{*} G(p) G(p)^{*} d \mu(p) .
$$

Since $F\left(a_{j}\right)^{*} \delta_{j}=0$ for all $j$, equation (5.13) gives

$$
0=\delta_{j}^{*} F\left(a_{j}\right) F\left(a_{j}^{*}\right) \delta_{j}=\int\left|\phi_{p}\left(a_{j}\right)\right|^{2}\left\|G(p)^{*} \delta_{j}\right\|^{2} d \mu(p) .
$$

Consequently, for each $j, \phi_{p}\left(a_{j}\right)^{*} G(p)^{*} \delta_{j}=0$ for almost every $p$, and so off of a set $Z_{0} \subset \Pi$ of measure zero, $\phi_{p}\left(a_{j}\right)^{*} G(p)^{*} \delta_{j}=0$ for all $p$ and each $j$. Thus, by defining $G(p)=0$ on $Z_{0}$, we may assume that equation (5.13) holds and

$$
\phi_{p}\left(a_{j}\right)^{*} G(p)^{*} \delta_{j}=0
$$

for all $p, j$.

Let $\Delta_{0}=\{p \in \Pi: G(p)=0\}$. If $p \notin \Delta_{0}$, then for each $j$, either $\phi_{p}\left(a_{j}\right)=0$ or $G_{p}^{*} \delta_{j}=0$. Since $G_{p}$ is a multiple of $\delta_{j_{1}(p)}$ and no three of the $\delta_{j}$ are collinear, it follows that $\phi_{p}$ has zeros at two of the $a_{j}$, say $a_{j_{1}(p)}, a_{j_{2}(p)}$, and the $\delta_{j_{3}(p)}$ and $\delta_{j_{4}(p)}$ are collinear (and orthogonal to $\delta_{j(p)}$ ), where $\left\{a_{1}, \ldots, a_{4}\right\}=\left\{a_{j_{1}(p)}, \ldots, a_{j_{4}(p)}\right\}$. We can now turn to Theorem 4.3 to conclude that $z_{1}(p)=a_{j_{1}(p)}, z_{2}(p)=a_{j_{2}(p)}$. In particular, $\phi_{p}$ has distinct zeros and $\delta_{j_{1}(p)}$ and $\delta_{j_{2}(p)}$ are collinear (and orthogonal to $\delta_{j_{3}(p)}$ and $\left.\delta_{j_{4}(p)}\right)$. Let $J_{1}=\left\{a_{j_{1}(p)}, a_{j_{2}(p)}\right\}$ and $J_{2}=\left\{a_{j_{3}(p)}, a_{j_{4}(p)}\right\}$. In addition, 
let $\Delta_{1}$ denote the one-dimensional subspace of $\mathbb{C}^{2}$ spanned by $\delta_{j_{1}(p)}$ and $\Delta_{2}$ the one-dimensional subspace of $\mathbb{C}^{2}$ spanned by $\delta_{j_{3}(p)}$.

If $p^{\prime} \notin \Pi_{0}$, then by arguing as above, either $G\left(p^{\prime}\right) \in \Delta_{1}$ or $G\left(p^{\prime}\right) \in \Delta_{2}$. In the former case, the zeros of $\phi_{p^{\prime}}$ are in $J_{2}$ and in the latter in $J_{1}$. Hence, for each $p$, either

(0) $G(p)=0$; or

(1) $G(p) \in \Delta_{1}$ and the nonzero zeros $z_{1}(p), z_{2}(p)$ are in $J_{2}$; or

(2) $G(p) \in \Delta_{2}$ and the nonzero zeros $z_{1}(p), z_{2}(p)$ are in $J_{1}$.

Let

$$
\begin{aligned}
& \Pi_{0}=\{p \in \Pi: \text { (0) holds }\}, \\
& \Pi_{1}=\{p \in \Pi: \text { (1) holds }\}, \\
& \Pi_{2}=\{p \in \Pi: \text { (2) holds }\} .
\end{aligned}
$$

If $p, q \in \Pi_{1}$, then $\phi_{p}$ and $\phi_{q}$ have the same zeros, and are therefore equal up to a rotation. Hence, for $p, q \in \Pi_{1}, \phi_{p}(z) \phi_{p}(w)^{*}=\phi_{q}(z) \phi_{q}(w)^{*}$. Choose $p^{1} \in \Pi_{1}$ and let $\psi_{1}=\phi_{p^{1}}$ denote a representative. If $\Pi_{2}$ is not empty, choose $p^{2} \in \Pi_{2}$ and let $\psi_{2}=\phi_{p^{2}}$. Otherwise, let $\psi_{2}=0$. Substituting into equation (5.12) and writing the integral as the sum of the integrals over $\Pi_{1}$ and $\Pi_{2}$ gives

$$
F(z) F(w)^{*}=h_{1} \psi_{1}(z) \psi_{1}(w)^{*} h_{1}+h_{2} \psi_{2}(z) \psi_{2}(w)^{*} h_{2}^{*}
$$

for some $h_{j} \in \Delta_{j}$. Substituting $z=w=1$ and using the fact that $F(1) F(1)^{*}=I$ shows that $\left\{h_{1}, h_{2}\right\}$ is an orthonormal basis for $\mathbb{C}^{2}$ (and $\psi_{2} \neq 0$ ). Thus, we can apply the previous lemma and conclude that $F$ is diagonalizable.

\section{THE OBSTRUCTION}

We now demonstrate a $2 \times 2$ matrix function $\Psi$ which is unitary on the boundary, analytic across the boundary, has a standard zero set, and which cannot be diagonalized. Since by Theorem 5.13, any function satisfying these conditions which has $\rho_{\Psi}=1$ is diagonalizable, $\rho_{\Psi}$ must be less than 1 for this $\Psi$ and so by Theorem 5.2 . rational dilation does not hold.

Recall the matrix inner functions $\Psi_{\eta, t}$ introduced in subsection 3.2. The $t$ was fixed at the outset of that section. By Lemma 3.8 for small $\eta, \Psi=\Psi_{\eta, t}$ has a standard zero set. Let $a_{j}^{0}, \delta_{j}^{0}$ denote the standard zero set for $\Psi_{0, t}$. (In particular, we can assume $\delta_{1}^{0}=\delta_{2}^{0}=e_{1}$ and $\delta_{3}^{0}=\delta_{4}^{0}=e_{2}$.) Let $\epsilon^{0}>0$ denote the $\epsilon>0$ in Theorem 4.3 corresponding to this zero set. Thus, for a small $\eta>0$ the function $\Psi=\Psi_{\eta, t}$ has a standard zero set and this zero set satisfies the conditions in Theorem 4.3. This $\Psi$ is the promised function.

Lemma 6.1. $\Psi$ has the following properties:

(1) $\Psi$ is unitary-valued on B;

(2) $\Psi$ has a standard zero set;

(3) the zero set $a_{1}, \ldots, a_{4}, \delta_{1}, \ldots, \delta_{4}$ has the property: if $h$ satisfies

$$
h(\zeta)=\sum_{1}^{4} c_{j} K^{0}\left(\zeta, a_{j}\right) \delta_{j}+v
$$

for some $c_{1}, \ldots, c_{4} \in \mathbb{C}$ and $v \in \mathbb{C}^{2}$ and if $h$ does not have a pole at either $P_{1}$ or $P_{2}$, then $h$ is constant; 
(4) $\Psi$ is not diagonalizable; i.e., there do not exist fixed unitaries $U$ and $V$ so that $U \Psi V^{*}$ is pointwise diagonal.

Proof. The only thing that remains to be proved is that $\Psi$ is not diagonalizable. We argue by contradiction. Suppose there is a diagonal function $D$ and fixed unitaries $U, V$ so that $D=U \Psi V^{*}$. Of course $D$ must be unitary-valued on $B$. In particular, $D(1)$ is unitary and so by multiplying on the left (or right) by $D(1)^{*}$, it may be assumed that $D(1)=I$. Since $\Psi(1)=I, V=U$.

Let $\varphi_{1}, \varphi_{2}$ denote the diagonal entries of $D$. Since $D$ is unitary-valued on $B$, both $\varphi_{1}$ and $\varphi_{2}$ are unimodular on $B$. Further, as $\operatorname{det}(\Psi)$ has 6 zeros and a function unimodular on $B$ has at least three zeros or is constant, we conclude that either both $\varphi_{1}$ and $\varphi_{2}$ have three zeros and thus take each value in $\{|z| \leq 1\}$ exactly three times in $X$, or one has six zeros and the other is a unimodular constant $\gamma$. This latter case cannot occur, since then

$$
0=\Psi(0)=U^{*}\left(\begin{array}{ll}
\gamma & 0 \\
0 & 0
\end{array}\right) U .
$$

From Lemma 3.7 $\Psi\left(q_{1}\right) e_{1}=e_{1}$. Thus $U e_{1}$ is an eigenvector for $D\left(q_{1}\right)$ corresponding to eigenvalue 1 , whence at least one of $\varphi_{j}\left(q_{1}\right)$ is 1 . Similarly, $U e_{2}$ is an eigenvector for $D\left(q_{1}^{*}\right)$ with eigenvalue 1 , and at least one of $\varphi_{j}\left(q_{1}^{*}\right)$ is 1 . Now, $D\left(q_{1}\right)$ cannot be a multiple of the identity, as otherwise both $\varphi_{1}\left(q_{1}\right)$ and $\varphi_{2}\left(q_{1}\right)$ equal 1 , in which case at least one of these two functions takes the value 1 at both $q_{1}$ and $q_{1}^{*}$. Therefore, without loss of generality, $U e_{1}=\lambda_{1} e_{1}$ and $U e_{2}=\lambda_{2} e_{2}$ for unimodular $\lambda_{1}$ and $\lambda_{2}$. Since $D$ is also diagonal, we can assume $\lambda_{j}=1$. Hence, $\Psi=D$.

From Lemma 3.7. $\Psi\left(q_{2}\right)\left(\eta e_{1}+\left(1-\eta^{2}\right)^{\frac{1}{2}} e_{2}\right)=\eta e_{1}+\left(1-\eta^{2}\right)^{\frac{1}{2}} e_{2}$ so that

$$
D\left(q_{2}\right)\left(\eta e_{1}+\left(1-\eta^{2}\right)^{\frac{1}{2}} e_{2}\right)=\eta \varphi_{1}\left(q_{2}\right) e_{1}+\left(1-\eta^{2}\right)^{\frac{1}{2}} \varphi_{2}\left(q_{2}\right) e_{2}
$$

Since $\eta \neq 0$, it follows that $\varphi_{j}\left(q_{2}\right)=1$. Similarly, $\varphi_{j}\left(q_{2}^{*}\right)=1$. As $q_{2} \neq q_{2}^{*}$, the function $\varphi_{j}$ takes the value 1 twice on $B_{2}$, a contradiction.

To prove our main theorem, simply note that the first three conditions of Lemma 6.1 imply, in view of Theorem 5.13, that if $\rho_{\Psi}=1$, then $\Psi$ is diagonal. Hence, it follows that $\rho_{\Psi}<1$ and this completes the proof.

\section{EXISTENCE of A FINITE-Dimensional EXAMPLE}

Let $\mathcal{A}$ denote the closure of $\mathcal{R}(X)$, the rational functions with poles off of $X$ as a subspace of $C(X)$. That is, $\mathcal{A}=\overline{\mathcal{R}(X)} \subset C(X)$. The algebra $\mathcal{A}$ is an (abstract) operator algebra with the family of matrix norms,

$$
\|F\|=\sup \{\|F(z)\|: z \in X\},
$$

for $F=\left(f_{j, \ell}\right) \in M_{n}(\mathcal{A})$ and where $\|F(z)\|$ is the matrix norm of the $n \times n$ matrix $F(z)$.

Given a finite subset $\Lambda \subset R$, let $I_{\Lambda}$ denote the ideal $\{f \in A: f(\lambda)=0$ for all $\lambda \in$ $\Lambda$ \} of $\mathcal{A}$. The quotient

$$
\mathcal{A}_{\Lambda}=\mathcal{A} / I_{\Lambda}
$$

inherits, in a canonical way, an operator algebra structure from $\mathcal{A}$. Namely,

$$
\left\|\pi_{\Lambda}(F)\right\|=\inf \left\{\|G\|: G \in M_{n}(\mathcal{A}) \text { and } \pi_{\Lambda}(G)=\pi_{\Lambda}(F)\right\} .
$$

Here $\pi_{\Lambda}$ denotes the quotient map. 
The following is a special case of a version of a theorem of Vern Paulsen [27. By a representation $\nu$ of $\mathcal{A}$ is meant a unital homomorphism $\nu: \mathcal{A} \rightarrow B(H)$, where $H$ is a Hilbert space. The representation $\nu$ is contractive if $\|\nu(f)\| \leq\|f\|$ for all $f \in F$ and is completely contractive if for each $n$ and each $F=\left(f_{j, \ell}\right) \in$ $M_{n}(\mathcal{A}),\|\nu(F)\|=\left\|\left(\nu\left(f_{j, \ell}\right)\right)\right\| \leq\|F\|$. Make similar definitions for representations, contractive representations, and completely contractive representations of $\mathcal{A}_{E}$.

Theorem 7.1 (Paulsen). Every contractive representation of $\mathcal{A}$ is completely contractive if and only if every contractive representation of every $\mathcal{A}_{\Lambda}$ is completely contractive.

The following observation of Paulsen is also useful. We give the proof.

Proposition 7.2. If $\nu: \mathcal{A}_{\Lambda} \rightarrow B(H)$ is contractive, but not completely contractive, then $\nu \circ \pi_{\Lambda}: \mathcal{A} \rightarrow B(H)$ is contractive, but not completely contractive.

Proof. Since $\nu$ and $\pi_{\Lambda}$ are contractive, the composition $\nu \circ \pi_{\Lambda}$ is contractive. On the other hand, if $\nu \circ \pi_{\Lambda}$ is completely contractive, then it follows from the definition of the norm on $M_{n}\left(\mathcal{A}_{E}\right)$ as the quotient norm, that $\nu$ is completely contractive. To see this, given $\pi_{\Lambda}(F)=\left(\pi_{\Lambda}\left(f_{j, \ell}\right)\right)$ in $M_{n}\left(\mathcal{A}_{E}\right)$ we can assume, by the definition of the quotient norm, that $F$ can be chosen so that $\|F\|$ is only a little larger than $\left\|\pi_{\Lambda}(F)\right\|$. Since $\nu \circ \pi_{\Lambda}$ is completely contractive, the norm of $\nu \circ \pi_{\Lambda}(F)$ is no larger than $\|F\|$ and the result follows.

We use Paulsen's results, together with our own, to argue that there is a matrix $X$ with $R$ as a spectral set, but which does not dilate to a normal operator with spectrum in $B$. It is enough to prove that there is a finite subset $\Lambda \subset R$, a finite-dimensional Hilbert space $H$, and a representation $\nu: \mathcal{A}_{E} \rightarrow B(H)$ which is contractive, but not completely contractive. We begin with a lemma.

Lemma 7.3. Fix $\Lambda$ a finite subset of $R$. If $\gamma: \mathcal{A}_{\Lambda} \rightarrow B(K)$ is a representation, then there exist subspaces $\mathcal{E}_{\lambda}, \lambda \in \Lambda$, of $K$ so that

(1) each $\mathcal{E}_{\lambda}$ is closed;

(2) $K$ is the algebraic direct sum $\hat{\bigoplus} \mathcal{E}_{\lambda}$; and

(3) if $f \in \mathcal{A}$ and $k \in \mathcal{E}_{\lambda}$, then

$$
\gamma\left(\pi_{\Lambda}(f)\right) k=\lambda k .
$$

Proof. For each $\lambda \in \Lambda$, choose a function $e_{\lambda} \in \mathcal{A}$ such that $e_{\lambda}(\mu)$ is 0 if $\mu \in \Lambda$, but $\mu \neq \lambda$ and such that $e_{\lambda}(\lambda)=1$. It follows that the operators $E_{\lambda}=\gamma\left(\pi_{\Lambda}\left(e_{\lambda}\right)\right)$ are idempotents such that $E_{\lambda} E_{\mu}=0$ if $\mu \neq \lambda$ and $\sum E_{\lambda}$ is the identity on $K$. Let $\mathcal{E}_{\lambda}=E_{\lambda} K$. Properties (1) and (2) are readily verified. As for (3),

$$
\begin{aligned}
\left(\gamma\left(\pi_{\Lambda}(f)\right)-f(\lambda)\right) E_{\lambda} & =\gamma\left(\pi_{\Lambda}(f-f(\lambda)) e_{\lambda}\right) \\
& =\gamma\left(\pi_{\Lambda}(0)\right)=0 .
\end{aligned}
$$

From our result, there is a representation of $\mathcal{A}$ which is contractive, but not completely contractive. From Paulsen's theorem, there exists a $\Lambda$ and Hilbert space $K$ (possibly infinite dimensional) and a representation $\gamma: \mathcal{A}_{\Lambda} \rightarrow B(K)$ which is contractive, but not completely contractive. Since this representation is not completely contractive, there exists an $n$ and $F \in M_{n}(\mathcal{A})$ such that

$$
1=\left\|\pi_{\Lambda}(F)\right\|<\left\|\gamma\left(\pi_{\Lambda}(F)\right)\right\| .
$$


Thus, there exists a vector

$$
x=\left(\begin{array}{c}
x_{1} \\
x_{2} \\
\vdots \\
x_{n}
\end{array}\right) \in \bigoplus_{1}^{n} K=\mathbb{C}^{n} \otimes K
$$

such that $\|x\|=1$ and

$$
\left\|\gamma\left(\pi_{\Lambda}(F)\right) x\right\|>1 .
$$

Write, $x_{j}=\sum x_{j}(\lambda)$ with respect to the algebraic direct sum $\hat{\oplus} \mathcal{E}_{\lambda}$ and let $\mathcal{H}$ denote the span of $\left\{x_{j}(\lambda): 1 \leq j \leq n, \quad \lambda \in \Lambda\right\}$. Thus, $\mathcal{H}$ is finite dimensional (the dimension is cardinality of $\Lambda$ times $n$ ). From condition (3) of our lemma, $\mathcal{H}$ is invariant for $\gamma$. Define $\nu: \mathcal{A}_{E} \rightarrow B(\mathcal{H})$ by restriction: $\nu(f)=\left.\gamma(f)\right|_{\mathcal{H}}$. Since also $x \in \mathbb{C}^{n} \otimes \mathcal{H}$ we have $\gamma\left(\pi_{\Lambda}(F)\right) x=\nu\left(\pi_{\Lambda}(F)\right) x$ and thus

$$
\left\|\nu\left(\pi_{\Lambda}(F)\right) x\right\|>1=\left\|\pi_{\Lambda}(F)\right\| .
$$

\section{REFERENCES}

1. M. B. Abrahamse and R. G. Douglas, A class of subnormal operators related to multiplyconnected domains, Advances in Math. 19 (1976), no. 1, 106-148. MR0397468 (53:1327)

2. Jim Agler, Rational dilation on an annulus, Ann. of Math. (2) 121 (1985), no. 3, 537-563. MR0794373 (87a:47007)

3. - On the representation of certain holomorphic functions defined on a polydisc, Topics in operator theory: Ernst D. Hellinger memorial volume, Oper. Theory Adv. Appl., vol. 48, Birkhäuser, Basel, 1990, pp. 47-66. MR 1207393 (93m:47013)

4. Jim Agler and John Harland, A monograph on function theory and Herglotz formulas for multiply connected domains.

5. Jim Agler, John Harland, and Benjamin Raphael, Classical function theory, operator dilation theory, and machine computations on multiply-connected domains.

6. Jim Agler and John E. McCarthy, Nevanlinna-Pick interpolation on the bidisk, J. Reine Angew. Math. 506 (1999), 191-204. MR1665697(2000a:47034)

7. _ Pick interpolation and Hilbert function spaces, Graduate Studies in Mathematics, vol. 44, American Mathematical Society, Providence, RI, 2002. MR1882259 (2003b:47001)

8. William Arveson, Subalgebras of $C^{*}$-algebras. II, Acta Math. 128 (1972), no. 3-4, 271-308. MR 0394232 (52:15035)

9. William B. Arveson, Subalgebras of $C^{*}$-algebras, Acta Math. 123 (1969), 141-224. MR0253059 (40:6274)

10. Joseph A. Ball and Kevin F. Clancey, Reproducing kernels for Hardy spaces on multiply connected domains, Integral Equations Operator Theory 25 (1996), no. 1, 35-57. MR.1386327 (97f:46042)

11. Joseph A. Ball and Tavan T. Trent, Unitary colligations, reproducing kernel Hilbert spaces, and Nevanlinna-Pick interpolation in several variables, J. Funct. Anal. 157 (1998), no. 1, 1-61. MR1637941 (2000b:47028)

12. Joseph A. Ball, Tavan T. Trent, and Victor Vinnikov, Interpolation and commutant lifting for multipliers on reproducing kernel Hilbert spaces, Operator theory and analysis (Amsterdam, 1997), Oper. Theory Adv. Appl., vol. 122, Birkhäuser, Basel, 2001, pp. 89-138. MR1846055 (2002f:47028)

13. Joseph A. Ball and Victor Vinnikov, Hardy spaces on a finite bordered Riemann surface, multivariable operator model theory and Fourier analysis along a unimodular curve, Systems, approximation, singular integral operators, and related topics (Bordeaux, 2000), Oper. Theory Adv. Appl., vol. 129, Birkhäuser, Basel, 2001, pp. 37-56. MR.1882690 (2003f:47016)

14. Stefan Bergman and Bruce Chalmers, A procedure for conformal mapping of triply-connected domains, Math. Comp. 21 (1967), 527-542. MR0228663 (37:4243a)

15. Kevin F. Clancey, Toeplitz operators on multiply connected domains and theta functions, Contributions to operator theory and its applications (Mesa, AZ, 1987), Oper. Theory Adv. Appl., vol. 35, Birkhäuser, Basel, 1988, pp. 311-355. MR1017675 (91f:47038) 
16. L Representing measures on multiply connected planar domains, Illinois J. Math. 35 (1991), no. 2, 286-311. MR1091446 (92e:46110)

17. John B. Conway, Functions of one complex variable, second ed., Graduate Texts in Mathematics, vol. 11, Springer-Verlag, New York, 1978. MR0503901 (80c:30003)

18. - The theory of subnormal operators, Mathematical Surveys and Monographs, vol. 36, American Mathematical Society, Providence, RI, 1991. MR1112128 (92h:47026)

19. H. M. Farkas and I. Kra, Riemann surfaces, second ed., Graduate Texts in Mathematics, vol. 71, Springer-Verlag, New York, 1992. MR1139765 (93a:30047)

20. John D. Fay, Theta functions on Riemann surfaces, Springer-Verlag, Berlin, 1973, Lecture Notes in Mathematics, Vol. 352. MR0335789 (49:569)

21. Stephen D. Fisher, Function theory on planar domains, Pure and Applied Mathematics, John Wiley \& Sons Inc., New York, 1983, A second course in complex analysis, A Wiley-Interscience Publication. MR0694693 (85d:30001)

22. Helmut Grunsky, Lectures on theory of functions in multiply connected domains, Vandenhoeck \& Ruprecht, Göttingen, 1978, Studia Mathematica, Skript 4. MR0463413 (573365)

23. Richard B. Holmes, Geometric functional analysis and its applications, Springer-Verlag, New York, 1975, Graduate Texts in Mathematics, No. 24. MR0410335 (53:14085)

24. David Mumford, Tata lectures on theta. I, Progress in Mathematics, vol. 28, Birkhäuser Boston Inc., Boston, MA, 1983, With the assistance of C. Musili, M. Nori, E. Previato and M. Stillman. MR 0688651 (85h:14026)

25. - Tata lectures on theta. II, Progress in Mathematics, vol. 43, Birkhäuser Boston Inc., Boston, MA, 1984, Jacobian theta functions and differential equations, With the collaboration of C. Musili, M. Nori, E. Previato, M. Stillman and H. Umemura. MR0742776 (86b:14017)

26. Zeev Nehari, Conformal mapping, Dover Publications Inc., New York, 1975, Reprinting of the 1952 edition. MR0377031 (51:13206)

27. Vern Paulsen, Private communication.

28. — Completely bounded maps and operator algebras, Cambridge Studies in Advanced Mathematics, vol. 78, Cambridge University Press, Cambridge, 2002. MR1976867 (2004c:46118)

29. Vern I. Paulsen, Completely bounded maps and dilations, Pitman Research Notes in Mathematics Series, vol. 146, Longman Scientific \& Technical, Harlow, 1986. MR0868472 (88h:46111)

30. Gilles Pisier, A polynomially bounded operator on Hilbert space which is not similar to a contraction, J. Amer. Math. Soc. 10 (1997), no. 2, 351-369. MR.1415321 (97f:47002)

31. Donald Sarason, The $H^{p}$ spaces of an annulus, Mem. Amer. Math. Soc. No. 56 (1965), 78. MR0188824 (32:6256)

32. V. L. Vinnikov and S. I. Fedorov, On the Nevanlinna-Pick interpolation in multiply connected domains, Zap. Nauchn. Sem. S.-Peterburg. Otdel. Mat. Inst. Steklov. (POMI) 254 (1998), no. Anal. Teor. Chisel i Teor. Funkts. 15, 5-27, 244. MR.1691394 (2000d:47031)

School of Mathematics and Statistics, Merz Court, University of Newcastle upon Tyne, Newcastle upon Tyne, NE1 7RU, United Kingdom

E-mail address: m.a.dritschel@ncl.ac.uk

Department of Mathematics, University of Florida, Box 118105, Gainesville, Florida 32611-8105

E-mail address: sam@math.ufl.edu 